\title{
Intermittent pressure overload triggers hypertrophy-independent cardiac dysfunction and vascular rarefaction
}

\author{
Cinzia Perrino, ${ }^{1}$ Sathyamangla V. Naga Prasad, ${ }^{1}$ Lan Mao, ${ }^{1}$ Takahisa Noma, ${ }^{1}$ Zhen Yan, ${ }^{1}$ \\ Hyung-Suk Kim, ${ }^{2}$ Oliver Smithies, ${ }^{2}$ and Howard A. Rockman ${ }^{1}$ \\ 'Duke University Medical Center, Durham, North Carolina, USA. ${ }^{2}$ University of North Carolina, Chapel Hill, Chapel Hill, North Carolina, USA.
}

\begin{abstract}
For over a century, there has been intense debate as to the reason why some cardiac stresses are pathological and others are physiological. One long-standing theory is that physiological overloads such as exercise are intermittent, while pathological overloads such as hypertension are chronic. In this study, we hypothesized that the nature of the stress on the heart, rather than its duration, is the key determinant of the maladaptive phenotype. To test this, we applied intermittent pressure overload on the hearts of mice and tested the roles of duration and nature of the stress on the development of cardiac failure. Despite a mild hypertrophic response, preserved systolic function, and a favorable fetal gene expression profile, hearts exposed to intermittent pressure overload displayed pathological features. Importantly, intermittent pressure overload caused diastolic dysfunction, altered $\beta$-adrenergic receptor ( $\beta A R$ ) function, and vascular rarefaction before the development of cardiac hypertrophy, which were largely normalized by preventing the recruitment of PI3K by $\beta$ AR kinase 1 to ligand-activated receptors. Thus stress-induced activation of pathogenic signaling pathways, not the duration of stress or the hypertrophic growth per se, is the molecular trigger of cardiac dysfunction.
\end{abstract}

\section{Introduction}

Cardiac hypertrophy is a universal response of the heart to injury or overload, which according to the Law of Laplace acts to normalize increased wall stress (1). Initially described as a beneficial and necessary response to maintain cardiac output under conditions of overload (1), hypertrophy has more recently been recognized as a risk factor for increased mortality (2). A large number of studies now collectively demonstrate that modulation of the hypertrophic growth of the heart is feasible without provoking hemodynamic compromise (3), despite increased wall stress (4). In both animal studies and clinical trials, inhibition of hypertrophic growth usually results in the amelioration of LV dysfunction (3-5). Therefore, a concept of continuous progression from compensated hypertrophy toward heart failure has recently led to proposing cardiac hypertrophy as an early therapeutic target $(3,6,7)$. However, the blockade of the growth response is not always beneficial (8-12), and importantly, inhibition of the growth response is not absolutely required to ameliorate cardiac dysfunction $(13,14)$. Moreover, hypertrophy is also a response of the heart to physiological stresses such as endurance exercise, but the athlete's heart does not progress to heart failure. Thus the precise role of cardiac hypertrophy in response to increased stress is largely undefined.

The mechanism by which the heart adapts to physiological or pathological loads producing an adaptive or a maladaptive pheno-

Nonstandard abbreviations used: $\beta$ AR, $\beta$-adrenergic receptor; $\beta$ ARK1, $\beta$ AR kinase 1 ; BW, body weight; CS, cell shortening; cTAC, chronic TAC; FS, fractional shortening; EDPVR, end-diastolic pressure-volume relationship; Ees, end-systolic elastance; GPCR, G protein-coupled receptor; ISO, isoproterenol; iTAC, intermittent TAC; iTAC $\gamma_{\text {inact }}$, cardiac-specific overexpression of catalytically inactive PI3K $\gamma ; \mathrm{LV} / \mathrm{BW}$, LV weight/BW; MHC, myosin heavy chain; MT, Masson trichrome; PI3K $\gamma_{\text {inact, }}$, catalytically inactive PI3K $\gamma$; SERCA2a, sarcoplasmic reticulum $\mathrm{Ca}^{++}$ATPase; TAC, transverse aortic constriction.

Conflict of interest: The authors have declared that no conflict of interest exists. Citation for this article: J. Clin. Invest. 116:1547-1560 (2006). doi:10.1172/JCI25397. type is currently unknown. Since most pathological causes are usually chronic while physiological stresses are intermittent by nature, it is thought likely that the duration of the stimulus could be critical in this differentiation. It is also possible that qualitatively different overloads are sensed differently by the heart, even if applied for the same period of time, producing divergent phenotypic responses.

In our current investigation, we hypothesized that the nature of the stress on the heart, rather than its duration, is a key determinant of the maladaptive phenotype. To test our hypothesis, we established a mouse model of intermittent pressure overload to apply a pathological stress for periods of duration identical to a swimming exercise protocol. Similar to physical training, intermittent pressure overload induced a mild hypertrophic response with no fetal gene reexpression. However, intermittent pressure overload induced the activation of signaling pathways that led to a pathological molecular, structural, and functional phenotype that could be rescued, at least in part, by genetic manipulation of the $\beta$-adrenergic receptor $(\beta A R)$ signaling pathway. Our results indicate that hypertrophy of the heart is a time-dependent reaction to cardiac stress that does not by itself lead to a pathological phenotype. Rather, the molecular signature of the overloaded heart, not the growth response per se, is the trigger that leads to cardiac adaptation or decompensation.

\section{Results}

The duration of stress on the heart determines the magnitude of LV bypertrophy development and the early deterioration of cardiac function. In this study, we used a number of mouse models to test the roles of duration and nature of the overload on the heart in the development of a maladaptive phenotype (experimental design I; Figure 1A). To induce physiological hypertrophy, we strenuously trained mice through forced swimming (15) or through voluntary running in wheel-equipped cages (16). To induce pathological hypertrophy, we used transverse aortic constriction (TAC) to impose chronic pressure overload on the heart (referred to as chronic 
A

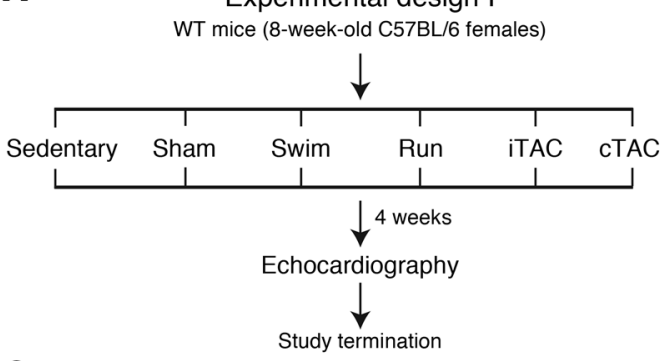

B
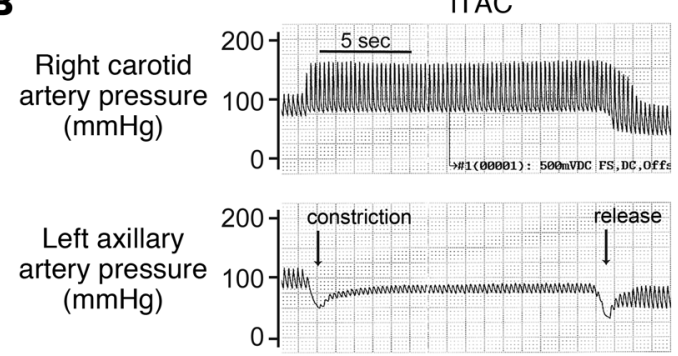

E

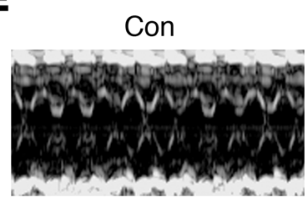

Swim

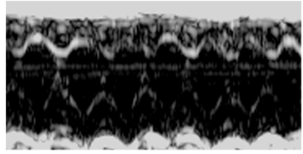

iTAC

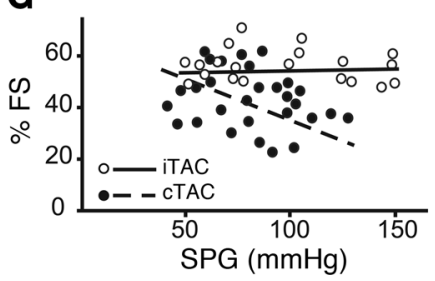

G

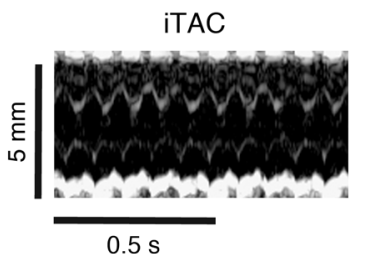

D

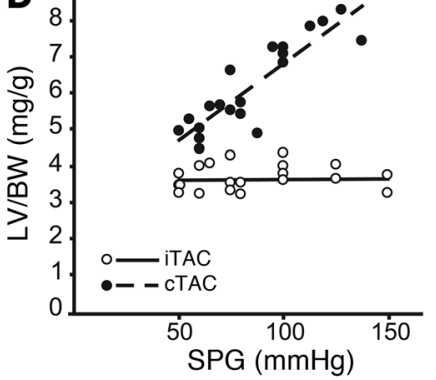

$\mathbf{F}$

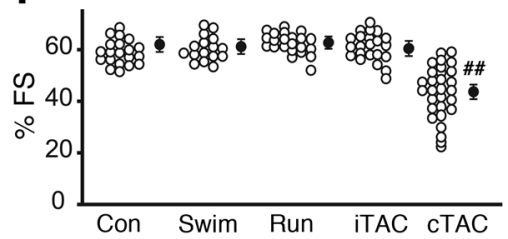

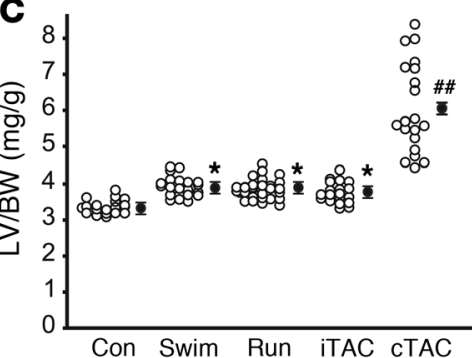

Co
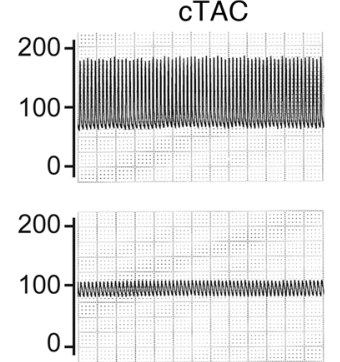

Figure 1

Duration of stress determines the magnitude of LV hypertrophy development. (A) Experimental design of the 4-week study involving different models of physiological hypertrophy (swimming or running) or pressure overload (chronic or intermittent). Sedentary and sham-operated mice were used as controls for swimming/running and iTAC/cTAC groups, respectively, and are shown as a single control group (Con). (B) Representative tracings showing the invasive measurement of arterial pressures in the right carotid and left axillary arteries in iTAC and cTAC mice. In all ITAC animals included in the study, pulling of the externalized suture caused a rapid increase in the right carotid systolic pressure, with or without a decrease in the left axillary systolic pressure that promptly regressed after release of the suture. (C) LV/BW ratios in mice from the different groups. Filled circles with error bars indicate average \pm SEM. ${ }^{*} P<0.05$ versus control; \#\# $P<0.01$ versus all other groups; ANOVA with Neuman-Keuls correction. (D) LV/BW ratios plotted against systolic pressure gradients measured at study termination in iTAC and cTAC mice. (E) Representative echocardiograms from the different groups of animals after 4 weeks of different protocols. (F) Percent FS in mice from the different groups. ${ }^{\#} P<0.01$ versus all other groups; ANOVA with Bonferroni correction. (G) Percent FS plotted against systolic pressure gradients (SPG) measured at study termination in iTAC and cTAC mice.

TAC; cTAC) as previously described (17), with some modifications (see Methods). In order to test the roles of quality and duration of the stress, we established a new mouse model to deliver the stress pressure, transiently and reversibly, for a duration equivalent to the physiological swimming protocol (see Supplemental Figure 1, $A$ and B; supplemental material available online with this article; doi:10.1172/JCI25397DS1). Briefly, to induce intermittent TAC (iTAC), a suture was placed around the transverse aorta and tunneled to the back of the mouse (see Methods and Supplemental Figure 1A). Sedentary mice and sham-operated animals were used as controls for the swimming/running and iTAC/cTAC groups, respectively, and since the individual data were indistinguishable their cumulated data were shown as 1 group.

To ensure the establishment of transient/reversible or chronic pressure overload, we invasively measured the trans-stenotic systolic pressure gradient in each TAC animal at the study's termination (Figure 1B). After 4 weeks, we measured the mass of cardiac chambers in all groups to determine the development of LV hypertrophy. As expect- ed, both exercise protocols led to a mild but significant increase in LV and heart weights normalized to body weight (BW; Table 1 and Figure 1C). Importantly, the amount of cardiac hypertrophy developed by iTAC mice was indistinguishable from swimming and running groups, in contrast to mice undergoing CTAC, which displayed a more pronounced hypertrophic growth response (Table 1 and Figure 1C). Although the reversible pressure gradient was not measured during each iTAC session and thus the actual amount of pressure overload is difficult to quantify, the mild hypertrophic response in the iTAC mice was independent of the trans-stenotic systolic pressure gradient measured at the time of study termination (Figure 1D).

We next evaluated the functional effects of cardiac enlargement in these different protocols by transthoracic echocardiography in conscious animals (Figure 1E and Table 1). Only cTAC hearts displayed initial deterioration of cardiac function after 4 weeks, as expressed by reduced percent fractional shortening (FS; Figure 1, E and F), despite a similar range of pressure gradients in intermittent and chronic TAC mice (Figure 1G). Taken together, these results sug- 
Table 1

Echocardiographic and morphometric evaluation of hypertrophic mouse hearts after 4 weeks (experimental design I)

\begin{tabular}{|c|c|c|c|c|c|}
\hline & Control & Swimming & Running & ITAC & cTAC \\
\hline Echocardiography & $n=15$ & $n=16$ & $n=19$ & $n=24$ & $n=29$ \\
\hline LVEDD (mm) & $2.7 \pm 0.1$ & $2.9 \pm 0.1^{A}$ & $2.9 \pm 0.1^{A}$ & $2.7 \pm 0.1$ & $2.9 \pm 0.1^{A}$ \\
\hline LVESD (mm) & $1.0 \pm 0.1$ & $1.2 \pm 0.1$ & $1.1 \pm 0.1$ & $1.1 \pm 0.1$ & $1.7 \pm 0.1^{B}$ \\
\hline IVS (mm) & $0.7 \pm 0.1$ & $0.7 \pm 0.1$ & $0.8 \pm 0.1$ & $0.9 \pm 0.1^{A}$ & $1.1 \pm 0.1^{\mathrm{A}}$ \\
\hline PW (mm) & $0.6 \pm 0.1$ & $0.6 \pm 0.1$ & $0.8 \pm 0.1$ & $0.9 \pm 0.1^{A}$ & $1.1 \pm 0.1^{\mathrm{A}}$ \\
\hline FS $(\%)$ & $61.5 \pm 1.5$ & $59.9 \pm 1.5$ & $61.8 \pm 1.1$ & $60.6 \pm 1.4$ & $43.4 \pm 1.8^{B}$ \\
\hline Vcfc (circ/s) & $4.4 \pm 0.1$ & $4.3 \pm 0.2$ & $4.8 \pm 0.2$ & $4.1 \pm 0.2$ & $3.2 \pm 0.1^{B}$ \\
\hline $\mathrm{HR}(\mathrm{bpm})$ & $643 \pm 10$ & $566 \pm 13^{B}$ & $622 \pm 9$ & $646 \pm 8$ & $636 \pm 7$ \\
\hline Organ morphometry & $n=22$ & $n=21$ & $n=25$ & $n=17$ & $n=23$ \\
\hline BW $(\mathrm{g})$ & $21.0 \pm 0.3$ & $19.2 \pm 0.3$ & $21.3 \pm 0.2$ & $21.0 \pm 0.6$ & $20.7 \pm 0.3$ \\
\hline $\mathrm{LA}(\mathrm{mg})$ & $2.8 \pm 0.1$ & $3.6 \pm 0.1$ & $3.2 \pm 0.1$ & $5.2 \pm 0.4^{A}$ & $8.7 \pm 1.2^{\mathrm{B}}$ \\
\hline LV/BW (mg/g) & $3.3 \pm 0.03$ & $3.9 \pm 0.06^{A}$ & $3.8 \pm 0.05^{A}$ & $3.7 \pm 0.07^{A}$ & $6.0 \pm 0.25^{B}$ \\
\hline $\mathrm{H} / \mathrm{BW}(\mathrm{mg} / \mathrm{g})$ & $4.6 \pm 0.03$ & $5.2 \pm 0.05^{A}$ & $5.1 \pm 0.05^{A}$ & $4.9 \pm 0.10^{A}$ & $7.4 \pm 0.31^{B}$ \\
\hline L/BW (mg/g) & $7.5 \pm 0.1$ & $7.4 \pm 0.3$ & $7.0 \pm 0.1$ & $8.4 \pm 0.5^{A}$ & $10.5 \pm 0.8^{B}$ \\
\hline Cellular morphometry & $n=6$ & $n=6$ & & $n=4$ & $n=8$ \\
\hline CM width $(\mu \mathrm{m})$ & $21.1 \pm 0.5$ & $23.4 \pm 0.9$ & - & $25.0 \pm 0.5^{A}$ & $25.1 \pm 0.9^{A}$ \\
\hline $\mathrm{CM}$ area $\left(\mu \mathrm{m}^{2}\right)$ & $2,286.7 \pm 61.5$ & $2,609.0 \pm 68.5^{A}$ & - & $2,599.7 \pm 68.8^{A}$ & $2,801.9 \pm 88.1^{A}$ \\
\hline
\end{tabular}

LVEDD, LV end-diastolic diameter; LVESD, LV end-systolic diameter; IVS, interventricular septum; PW, posterior wall; Vcfc, ventricular circumferential shortening, corrected by heart rate; circ/s, circumferential shortening per second; HR, heart rate; LA, left atrium weight; H/BW, heart weight/BW; L/BW, lung weight/BW; CM, cardiomyocyte. ${ }^{A} P<0.01$ versus control. ${ }^{B} P<0.001$ versus all other groups. Comparisons were made using ANOVA with Neuman-Keuls correction for echocardiography and organ morphometry and Student's $t$ test with Bonferroni correction for cellular morphometry. ment groups compared with sedentary and sham-operated control mice, with no differences among the treatment groups (Figure 2A).

Physiologically-applied pressure overload induces a physiological gene expression profile but a pathological structural phenotype. In contrast to physiological hypertrophy, pathological hypertrophy is consistently associated with the reexpression of fetal genes $(21,22)$. In order to determine the gene expression signature of iTAC mice, we carried out RT-PCR studies for selected genes from hearts of control, swimming, iTAC, and cTAC mice $(n=5$ per group). Overall, iTAC mice revealed a gene expression profile that was more similar to swimming mice than to cTAC mice (Figure 2, $B$ and $C)$. In particular, $\beta$ and gest that chronic pressure overload produces a greater hypertrophic response and a deterioration in cardiac function compared with intermittently applied pressure overload. Although it is possible that other factors might contribute to the pathological phenotype in cTAC mice, such as aortic root dilatation or activation of local or systemic inflammatory processes, we never detected abnormalities in cardiac function in the absence of a chronic pressure gradient.

The increase in endogenous catecholamine levels has a primary role in the development of cardiac hypertrophy following chronic pressure overload (18), but it is also required to obtain the physiological adaptations that occur during exercise $(19,20)$. We have previously shown that chronic pressure overload produces an increase in renal renin transcription that is mainly dependent on $\beta A R$ stimulation of renal juxtaglomerular cells, since it is abolished in pressure-overloaded mice lacking endogenous catecholamines (18). We therefore measured renal mRNA levels of renin by real-time RTPCR (18) to evaluate the level of chronic sympathetic nervous system activation in the different groups of treated mice. Interestingly, steady-state renal renin mRNA was similarly increased in the treat-

\section{Figure 2}

Chronic pathological stress is required to induce the fetal gene expression reprogramming. (A) Gene expression analysis of renal renin in kidneys of swimming, running, iTAC, and CTAC mice by RT-PCR. Data are shown as fold change over control \pm SEM. Differences were not significant. (B and $\mathbf{C}$ ) Gene expression analysis of $\alpha$ and $\beta$ isoforms of $\mathrm{MHC}$, atrial natriuretic peptide (ANP), brain natriuretic peptide (BNP), angiotensin II type 1a receptor (ATR), adrenomedullin (AM) and natriuretic peptide receptor A (NPRA) in hearts of swimming, iTAC, and cTAC mice by real-time RT-PCR of LV mRNA. Sedentary animals were used as controls for swimming mice; sham-operated animals were used for iTAC or ITAC. Data are shown as fold change over control \pm SEM. $\# P<0.05$ versus swimming; $¥ P<0.01$ versus iTAC; Student's $t$ test. $\alpha$ myosin heavy chain (MHC) genes in iTAC hearts displayed a pattern of regulation more closely resembling physiologic hypertrophy than the cTAC hearts, as exemplified by a lower $\beta \mathrm{MHC} / \alpha \mathrm{MHC}$

A

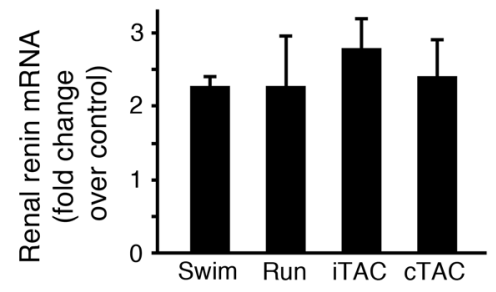

B
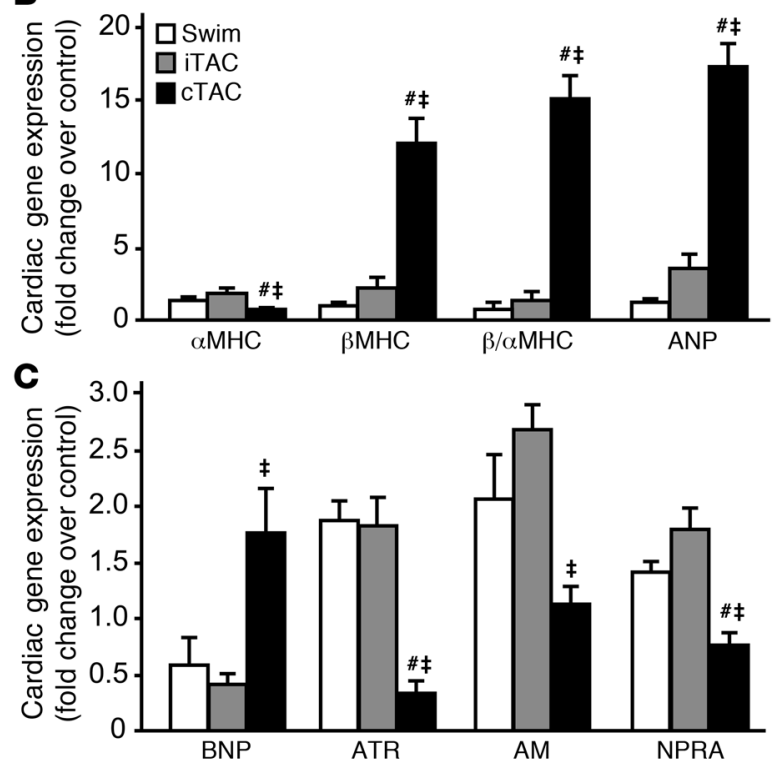
A
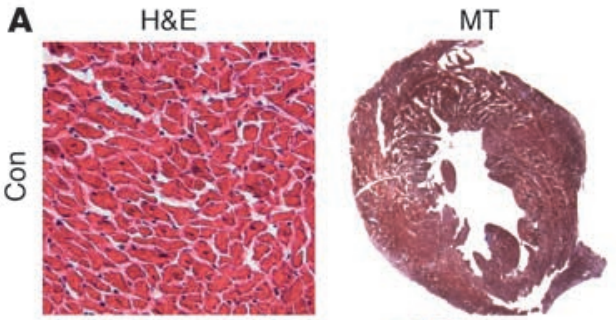

है
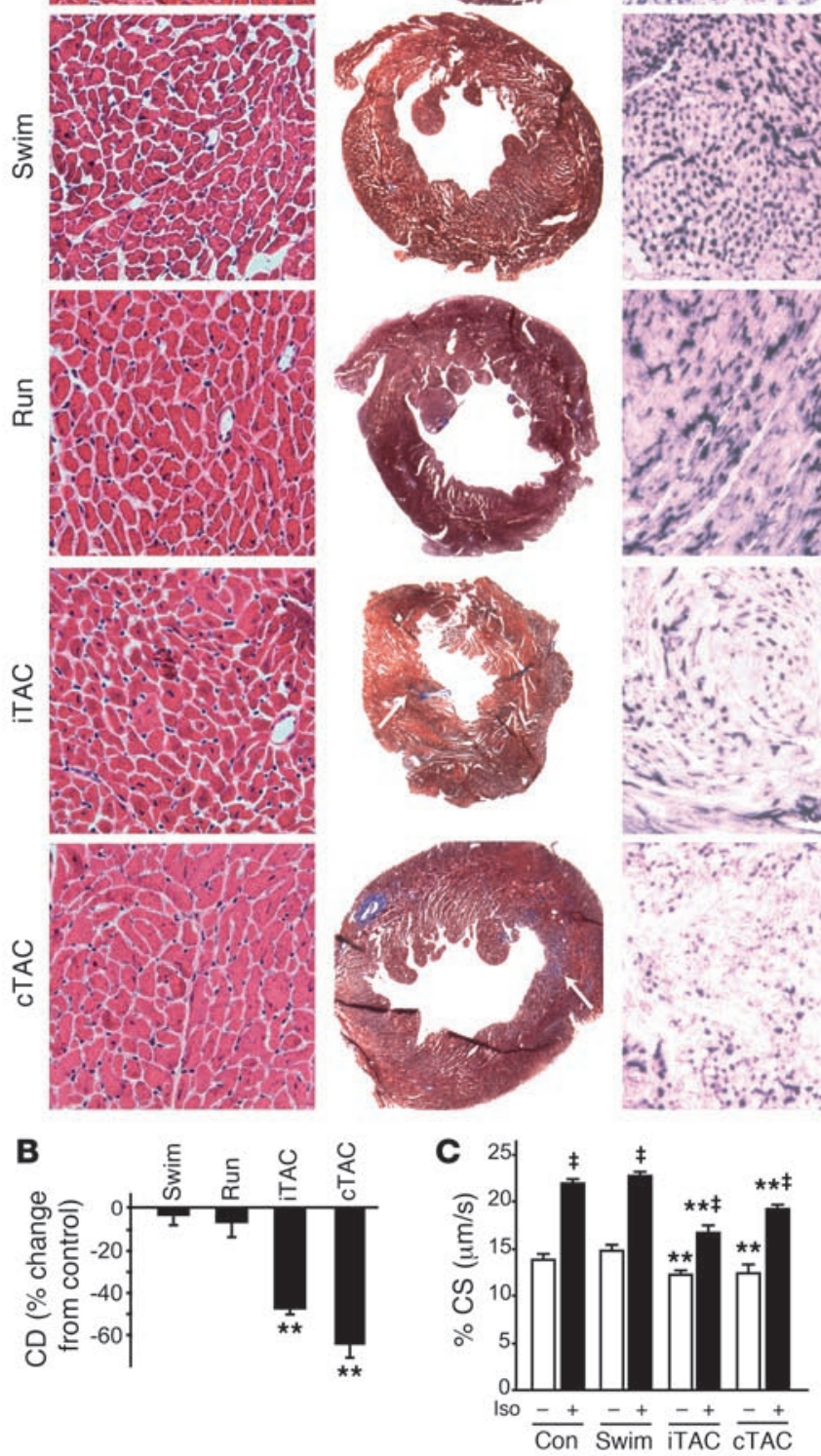

CD
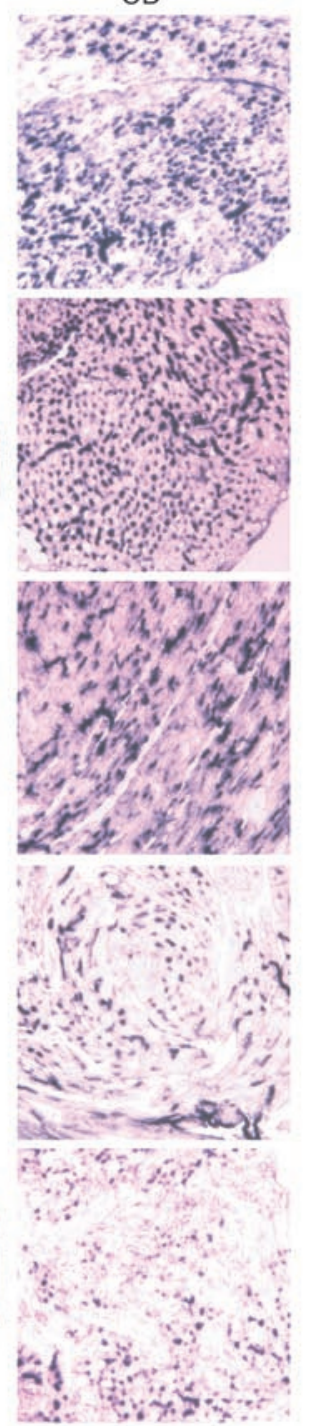

B

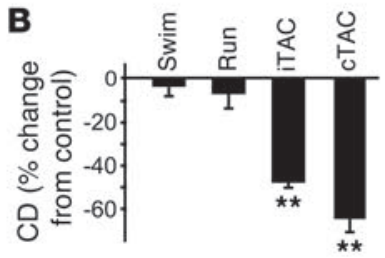

Figure 3

Hearts exposed to physiologically applied pressure overload display a pathological structural and cellular phenotype. (A) Representative staining of cardiac sections with H\&E (magnification, $\times 400$ ), MT (magnification, $\times 20$ ), and endothelial alkaline phosphatase (to determine capillary density, CD; magnification, $\times 400$ ). (B) Quantification of capillary density in cardiac sections stained for endothelial alkaline phosphatase expressed as percent change from control. ${ }^{\star \star} P<0.01$ versus control; ANOVA with Bonferroni correction. (C) Summary data for contractility studies of cardiac cells isolated from hearts of control, swimming, ITAC, and cTAC mice after 4 weeks of training ( $n=5-10$ mice per group; in each heart, $10-15$ cells were analyzed under basal conditions and following ISO stimulation). Percent CS under basal conditions (white bars) and following ISO stimulation (black bars) is shown. $¥ P<0.01$ versus respective basal group; ${ }^{\star \star} P<0.01$ versus corresponding control and swimming groups; ANOVA with Neuman-Keuls correction.

and isolated cardiomyocytes (Figure 1 and Table 1). Masson trichrome (MT) staining revealed little fibrosis in iTAC hearts $(2.2 \% \pm 0.3 \%$ of the total area), which was significantly less than in cTAC hearts $(5.5 \% \pm 2.0 \%$ of the total area; Figure $3 \mathrm{~A}$, middle panels). Fibrosis, a typical signature of pathological cardiac hypertrophy, was absent in the hearts of mice in the swimming and running groups (Figure 3A, middle panels). Remarkably, despite the seemingly physiological phenotype, we found that iTAC hearts displayed a marked and diffuse reduction of capillary density that was nearly equivalent to the loss in capillaries in cTAC hearts (Figure 3A, right panels, and Figure 3B). The marked loss in capillary density determined by staining for endothelial alkaline phosphatase was confirmed by staining for the endothelial marker CD31 (data not shown). Importantly, capillary density was completely preserved in hearts of exercising mice (Figure 3, A and B).

The marked loss of capillary density in iTAC hearts is in stark contrast to the findings of preserved global systolic function and a gene expression profile considered to be physiologic. To precisely determine the intrinsic contractile properties of cardiac cells exposed to the different overloads, we measured basal and $\beta$-agonist-stimulated cell contractility in cardiomyocytes from the hearts of control, swimming, iTAC, and CTAC mice. In cells from iTAC hearts, basal contractility expressed by percent cell shortening (CS) was significantly reduced $(76.8 \% \pm 1.4 \%$ of control; $P<0.05$ ), as was the contractile response to isoproterenol (ISO) stimulation (iTAC, $1.3 \pm 0.06$ fold change over basal CS; control, $1.6 \pm 0.05$ fold change over basal CS; $P<0.05$; Figure $3 C)$. Moreover, cells from iTAC hearts displayed a slower rate of relaxation under both basal and ISO-stimulated conditions (Supplemental Figure 2A). To investigate the possible mechanisms underlying the abnormal relaxation in cells exposed to intermittent pressure overload, we measured the levels of sarcoplasmic reticulum $\mathrm{Ca}^{++}$ATPase (SERCA2a) and phospholamban, molecules involved in regulating $\mathrm{Ca}^{++}$reuptake, by immunoblotting. SERCA2a protein levels were selectively reduced in hearts exposed to pressure overload, either chronic or intermittent, while total protein levels and phosphorylation of its regulator phospholamban at Ser16 were unaffected (Supplemental Figure 2B). Consistent with the findings of abnormal cellular diastolic dysfunction, iTAC mice also displayed a significant increase of left atrium weight (Table 1), consistent with the increased afterload in these animals, and of lung weight $/ \mathrm{BW}$ ratios, most likely secondary to lung edema (Table 1). 
A

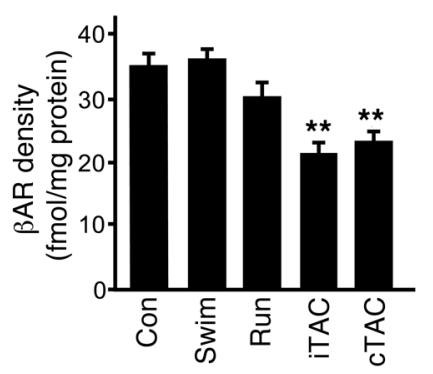

D IP: $\beta$ ARK1

Assay: PI3K activity

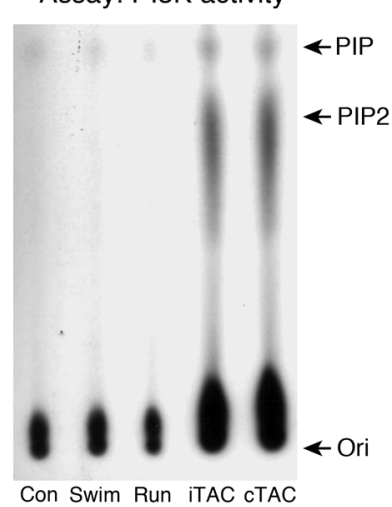

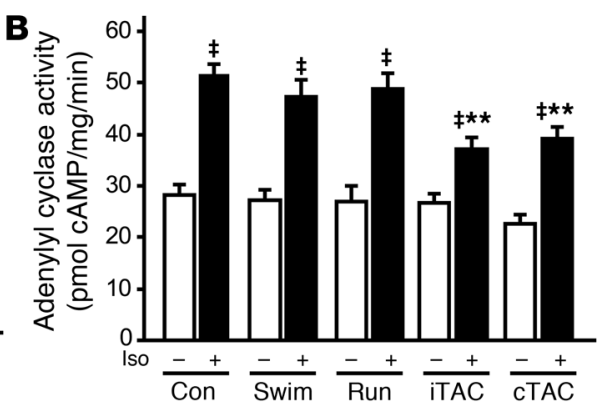

\section{C}

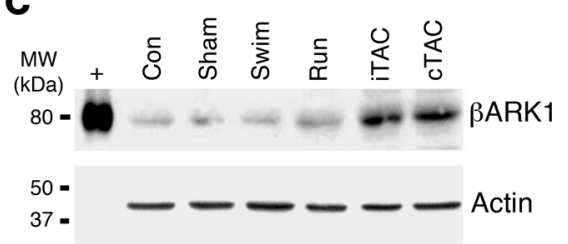

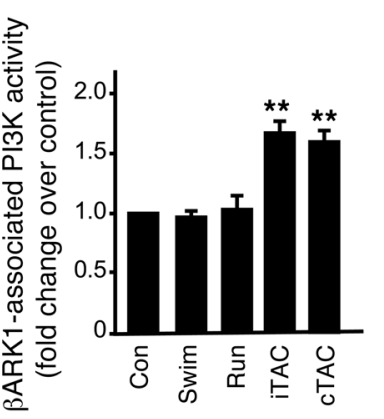

$\mathbf{E}$
$\mathbf{F}$

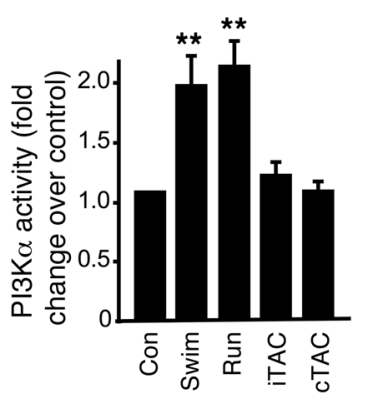

Figure 4

$\beta A R$ dysfunction is an early molecular sensor of pathological pressure overload. (A) $\beta$ AR density among membranes from hearts of control $(n=17)$, swimming $(n=7)$, running $(n=9)$, iTAC $(n=7)$, and cTAC $(n=11)$ mice. ${ }^{* *} P<0.01$ versus control. (B) Adenylyl cyclase activity at basal levels (white bars) and upon ISO stimulation (black bars) in hearts of control $(n=15)$, swimming $(n=9)$, running $(n=7)$, iTAC ( $n=6)$, and cTAC $(n=9)$ mice. ${ }^{\ddagger} P<0.01$ versus respective basal group; ${ }^{* \star} P<0.01$ versus control ISO; ANOVA with Bonferroni correction. (C) Myocardial lysates $(80 \mu \mathrm{g})$ of hearts from control, swimming, running, iTAC, and cTAC mice, along with the purified protein (+), were immunoblotted for $\beta A R K 1$. (D) Representative PI3K assay showing $\beta A R K 1$-associated PI3K activity in membrane lysates from hearts of control, swimming, running, iTAC, and cTAC mice (left panel). Summary data for all the experiments performed ( $n=4-7$ hearts per group) are shown at right. (E and F) Summary data for PI3K $\gamma(E)$ and PI3Ka activity $(\mathrm{F})$ in cytosolic lysates from the same hearts. Ori, origin; PIP, phosphatidylinositol monophosphate; PIP2, phosphatidylinositol bisphosphate. ${ }^{* \star} P<0.01$ versus control; Student's $t$ test with Bonferroni correction.

Taken together, these results show that despite a mild hypertrophic response, preserved global cardiac function, and a fetal gene expression profile considered to be favorable, intermittent pressure overload leads to abnormal cardiac morphology and cellular dysfunction. Furthermore, the blunted contractile responsiveness to $\beta A R$ stimulation suggests that abnormalities in $\beta A R$ system likely exist in iTAC hearts.

Pressure-overloaded hearts are characterized by $\beta A R$ dysfunction. Our previous studies have shown that desensitization and downregulation of $\beta A R$ s occur before the development of overt cardiac dysfunction in mice $(23,24)$. However, whether abnormalities in the $\beta$ AR system are intrinsically linked to the development of the pathological phenotype is controversial (3). In order to test the integrity of $\beta$ AR function following physiologically applied pressure overload, we measured $\beta A R$ density and adenylyl cyclase activity in the membrane fractions from control and hypertrophic hearts. $\beta A R$ levels were significantly reduced only in cTAC and iTAC mice (Figure 4A), resulting in reduced ISO-stimulated generation of cAMP (Figure $4 B$ ). Since previous studies have shown that the $\beta A R$ kinase 1 ( $\beta$ ARK1) is critically involved in $\beta$ AR dysfunction in pressure overload hypertrophy (25), we measured $\beta A R K 1$ protein levels in all groups of animals. A marked increase in $\beta A R K 1$ levels was found only in hearts exposed to either chronic or intermittent pressure overload (Figure 4C).

$\beta A R K 1$ associates with multiple isoforms of the enzyme PI3K to form a stable cytosolic complex that is recruited to activated $\beta$ ARs following prolonged receptor stimulation $(13,26,27)$. Increased membrane-targeted PI3K activity is associated with $\beta$ AR dysfunction in models of heart failure (13). We found a marked increase in $\beta A R K 1$-associated PI3K activity in cardiac membranes only in iTAC and CTAC hearts (Figure 4D). iTAC mice also displayed selective activation of PI3K $\gamma$, similar to cTAC (Figure 4E), whereas - consistent with previous studies (15) - we found a significant cardiac increase in PI3K $\alpha$ activity only in the swimming and running groups (Figure 4F). Taken together, these data demonstrate that qualitatively different overloads are uniquely sensed and transduced in the heart in a time-independent fashion. Moreover, our data suggest that $\beta \mathrm{AR}$ abnormalities, driven by increased levels of $\beta A R K 1$ and the membrane targeting of PI3K, occur in response to pressure overload, even when applied intermittently analogous to physiological stress.

Increased mechanical stress in pressure-overloaded hearts. Despite being exposed to stress for an identical period of time, exercising and intermittent pressure-overloaded hearts exhibited divergent structural and molecular features. To address the role of mechanical 
A

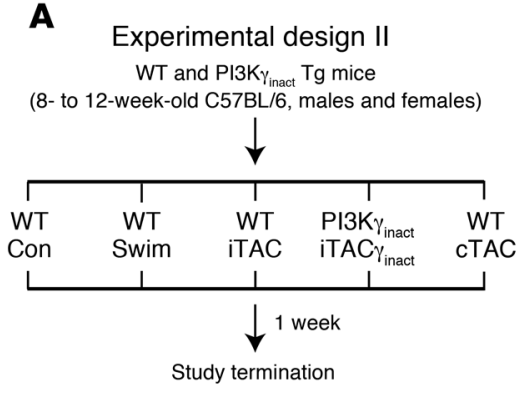

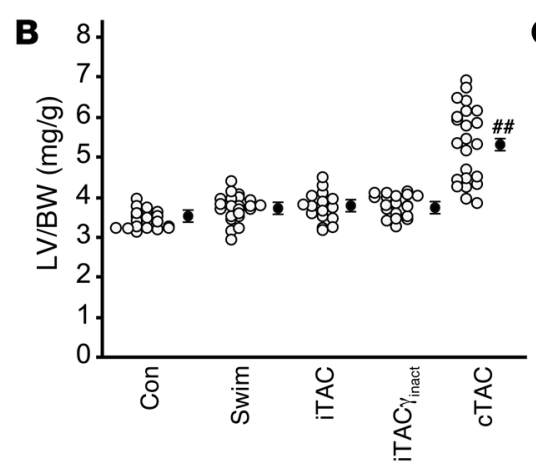

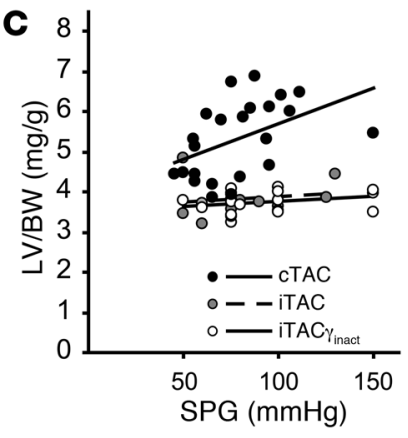

D

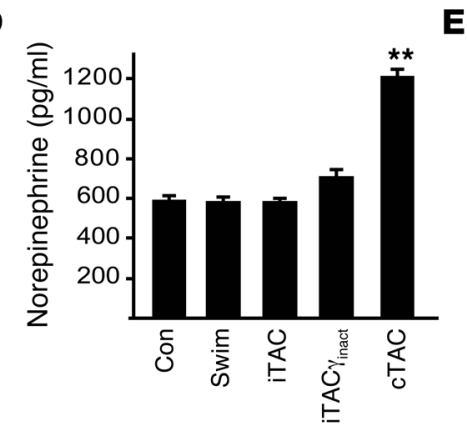

E
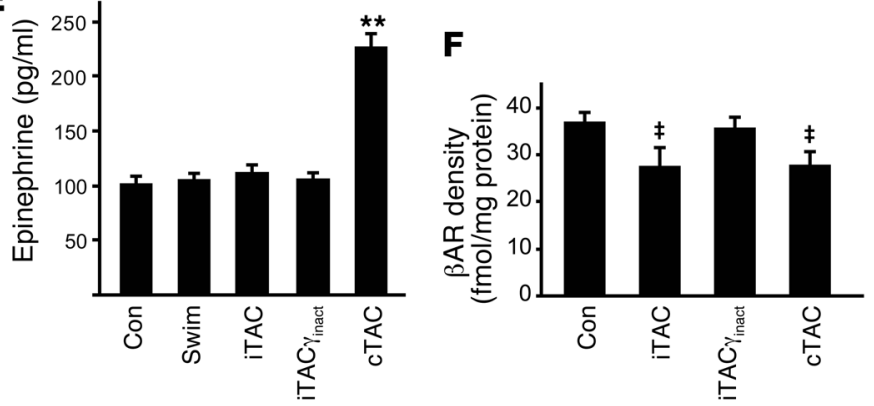

Figure 5

Transgenic inhibition of $\beta$ AR-targeted PI3K activity preserves $\beta A R$ signaling upon intermittent pressure overload. (A) Experimental design of the 1 -week study involving WT and PI3K $\gamma_{\text {inact }}$ transgenic mice. (B) LV/BW ratios in mice from the different groups. \#\# $P<0.01$ versus all other groups; ANOVA with Bonferroni correction. Filled circles with error bars indicate average \pm SEM. (C) LV/BW ratios plotted against respective systolic pressure gradients measured at study termination in iTAC, iTAC $\gamma_{\text {inact }}$, and cTAC mice. (D and E) Plasma levels of catecholamines norepinephrine (D) and epinephrine (E) in the different groups. ${ }^{* *} P<0.01$ versus control. (F) $\beta$ AR density among membranes from hearts of control $(n=12)$, $\operatorname{iTAC}(n=10), \operatorname{iTAC} \gamma_{\text {inact }}(n=9)$, and cTAC $(n=11)$ mice. ${ }^{\ddagger} P<0.01$ versus control and iTAC $\gamma_{\text {inact }} ;$ ANOVA with Bonferroni correction.

stress in the induction of the pathological phenotype, we evaluated the regulation of molecules involved in the signal transduction of mechanical signals. Plasma membrane levels of integrin $\beta_{1 \mathrm{D}}$, a well-known mechanotransducer, were significantly increased only in hearts of running and pressure-overloaded mice (Supplemental Figure 3A). Similarly, there was no discernable activation of the stress-activated kinase family (ERK, p38, and JNK1) upon swimming training, while these kinases were induced by chronic or intermittent pressure overload (Supplemental Figure 3, B-D). Interestingly, hearts from running mice showed significant activation of ERK and p38, but not JNK1 (Supplemental Figure 3, B-D). These differences in the hearts of mice between the swimming and running groups might possibly be attributed to the duration of the voluntary running, which was markedly longer than the forced swimming training (on average, $11.4 \pm 0.4 \mathrm{~h} / \mathrm{d}$ in running versus $3 \mathrm{~h} / \mathrm{d}$ in swimming mice), or to the shorter interval between termination of exercise and sacrifice (at least 12 hours for swimming and ITAC mice; $2-5$ hours for running mice). The differential activation of JNK1 between the hearts of running and TAC mice suggests a preferential role for JNK1 in pathological hypertrophy $(28$, 29) and indicates that pathological and physiological stresses are differently sensed and transduced by the heart.

Transgenic inbibition of $\beta A R$-targeted PI3K activity preserves $\beta A R$ signaling upon intermittent pressure overload. Our studies identified that one of the earliest molecular abnormalities induced by intermittent pressure overload on the heart is $\beta$ AR downregulation and desensitization. However, whether $\beta$ AR abnormalities are linked to the initiation of the pathological cardiac phenotype is unknown (3). To determine whether abnormalities in $\beta$ AR function can be experi- mentally dissected from the growth response induced by pressure overload and to test whether normalizing $\beta A R$ signaling is associated with rescue of the hypertrophy-independent pathological phenotype induced by iTAC, we applied intermittent pressure overload for only 7 days in WT mice and transgenic mice with cardiac-specific overexpression of catalytically inactive PI $3 \mathrm{~K} \gamma$ (iTAC $\gamma_{\text {inact }}$ mice; Figure 5A and Supplemental Figure 1C). We have previously shown that overexpression of catalytically inactive PI3K $\gamma$ (PI3K $\left.\gamma_{\text {inact }}\right)$ preserves $\beta A R$ function in a number of models of heart failure $(13,26,27)$.

After 1 week, neither swimming nor iTAC protocols resulted in a significant hypertrophic growth response (Figure 5B) or an increase in total cardiomyocyte area (cross-sectional area, control, 2,771 \pm 73 $\mu \mathrm{m}^{2}$; swimming, 2,841 $\pm 127 \mu \mathrm{m}^{2}$; iTAC, 2,665 $\pm 60 \mu \mathrm{m}^{2}$ ) despite a mild increase in the wall thickness in iTAC mice (Table 2). Only mice exposed to chronic pressure overload exhibited a significant increase in $\mathrm{LV}$ mass as shown by $\mathrm{LV}$ weight/BW (LV/BW) ratios (Figure $5 \mathrm{~B}$ ), which was positively correlated to the trans-stenotic systolic pressure gradient (Figure 5C). Importantly, PI3K $\gamma_{\text {inact }}$ overexpression did not affect the hypertrophic response induced by 1 week of intermittent pressure overload (Figure 5B and Table 2).

In order to measure the amount of sympathetic activation induced by these different stresses, we measured the resting levels of catecholamines in the different groups. After 1 week, only cTAC mice showed a significant increase in the levels of norepinephrine and epinephrine, while iTAC mice, similar to swimming mice, displayed low steady-state levels of catecholamines (Figure 5, D and E). However, cardiac membranes from iTAC hearts showed increased $\beta A R K 1$ levels and $\beta A R K 1$-associated PI3K activity (Supplemental Figure 4, A and B), resulting in downregulation 
Table 2

Echocardiographic and hemodynamic parameters in mice after 1 week of treatment (experimental design II)

\begin{tabular}{|c|c|c|c|c|c|}
\hline & $\begin{array}{l}\text { Control } \\
(n=11)\end{array}$ & $\begin{array}{l}\text { Swimming } \\
\quad(n=9)\end{array}$ & $\begin{array}{l}\text { iTAC } \\
(n=9)\end{array}$ & $\begin{array}{c}\text { iTAC } \gamma_{\text {inact }} \\
(n=9)\end{array}$ & $\begin{array}{c}\text { cTAC } \\
(n=15)\end{array}$ \\
\hline \multicolumn{6}{|l|}{ Echocardiography } \\
\hline $\mathrm{HR}(\mathrm{bpm})$ & $676 \pm 9$ & - & $630 \pm 9 A$ & $674 \pm 9$ & $657 \pm 7$ \\
\hline LVEDD (mm) & $2.6 \pm 0.05$ & - & $2.6 \pm 0.06$ & $2.6 \pm 0.04$ & $2.8 \pm 0.08 \mathrm{~A}$ \\
\hline IVS (mm) & $0.7 \pm 0.02$ & - & $0.9 \pm 0.04^{A}$ & $0.9 \pm 0.02^{\mathrm{A}}$ & $1.0 \pm 0.08^{A}$ \\
\hline PW (mm) & $0.6 \pm 0.01$ & - & $0.8 \pm 0.03^{A}$ & $0.8 \pm 0.03^{A}$ & $0.9 \pm 0.06^{A}$ \\
\hline FS $(\%)$ & $63.9 \pm 1.3$ & - & $64.9 \pm 1.6$ & $67.0 \pm 1.1$ & $52.2 \pm 2.5^{A}$ \\
\hline \multicolumn{6}{|c|}{ Pressure-volume loop analysis } \\
\hline $\mathrm{HR}(\mathrm{bpm})$ & $316 \pm 17$ & $298 \pm 8$ & $292 \pm 14$ & $321 \pm 28$ & $287 \pm 11$ \\
\hline $\mathrm{ESV}(\mu \mathrm{l})$ & $15.4 \pm 2.2$ & $16.7 \pm 1.6$ & $21.5 \pm 3.0$ & $12.9 \pm 1.0$ & $19.4 \pm 2.1$ \\
\hline EDV $(\mu l)$ & $36.8 \pm 2.2$ & $37.8 \pm 1.9$ & $39.8 \pm 3.1$ & $37.9 \pm 3.2$ & $38.2 \pm 2.6$ \\
\hline $\mathrm{Ea}(\mathrm{mmHg} / \mu \mathrm{l})$ & $3.9 \pm 0.3$ & $3.6 \pm 0.4$ & $4.3 \pm 0.8$ & $3.2 \pm 0.2$ & $3.6 \pm 0.3$ \\
\hline \multicolumn{6}{|l|}{ Systolic function } \\
\hline Max. press. (mmHg) & $96.7 \pm 3.2$ & $89.9 \pm 5.5$ & $81.3 \pm 5.0$ & $98.1 \pm 6.4$ & $89.7 \pm 3.2$ \\
\hline $\mathrm{ESP}(\mathrm{mmHg})$ & $89.8 \pm 3.9$ & $83.5 \pm 6.0$ & $75.0 \pm 4.8$ & $87.9 \pm 5.9$ & $76.2 \pm 3.4$ \\
\hline$d P / d t_{\max }(\mathrm{mmHg} / \mathrm{s})$ & $7,011 \pm 333$ & $5,979 \pm 309$ & $4,025 \pm 234^{A}$ & $4,532 \pm 215^{A}$ & $5,275 \pm 280^{\mathrm{A}}$ \\
\hline $\mathrm{EF}(\%)$ & $65.0 \pm 3.3$ & $60.2 \pm 1.7$ & $50.8 \pm 5.8$ & $67.8 \pm 1.9^{\mathrm{B}}$ & $56.6 \pm 3.8$ \\
\hline SV $(\mu \mathrm{l})$ & $24.2 \pm 1.6$ & $23.7 \pm 0.8$ & $21.2 \pm 3.1$ & $28.2 \pm 2.3$ & $23.6 \pm 1.9$ \\
\hline $\mathrm{CO}(\mathrm{ml} / \mathrm{min})$ & $7.6 \pm 0.6$ & $7.1 \pm 0.3$ & $6.1 \pm 0.9$ & $8.3 \pm 0.7^{B}$ & $6.5 \pm 0.6$ \\
\hline Ees $(\mathrm{mmHg} / \mu \mathrm{l})$ & $4.1 \pm 0.4$ & $3.8 \pm 0.4$ & $6.5 \pm 0.8^{A}$ & $5.3 \pm 0.5$ & $8.0 \pm 0.9^{A}$ \\
\hline \multicolumn{6}{|l|}{ Diastolic function } \\
\hline $\mathrm{EDP}(\mathrm{mmHg})$ & $8.2 \pm 1.3$ & $7.8 \pm 0.7$ & $18.7 \pm 3.0^{A}$ & $12.8 \pm 2.3$ & $13.9 \pm 1.2^{A}$ \\
\hline$d P / d t_{\min }(\mathrm{mmHg} / \mathrm{s})$ & $-5,571 \pm 201$ & $-4,750 \pm 282$ & $-3,259 \pm 426^{A}$ & $-3,324 \pm 271^{A}$ & $-3,292 \pm 260^{A}$ \\
\hline$\tau$ Glantz (ms) & $22.6 \pm 0.7$ & $25.2 \pm 1.2$ & $33.0 \pm 2.8^{A}$ & $21.7 \pm 1.7^{\mathrm{B}}$ & $38.5 \pm 3.8^{A}$ \\
\hline EDPVR $(\mathrm{mmHg} / \mu \mathrm{l})$ & $0.5 \pm 0.1$ & $0.5 \pm 0.1$ & $1.4 \pm 0.3^{A}$ & $0.4 \pm 0.1^{B}$ & $0.9 \pm 0.1^{A}$ \\
\hline
\end{tabular}

ESV, end-systolic volume; EDV, end-diastolic volume; Ea, elastic elastance; Max. press., maximum pressure; ESP, end-systolic pressure; EF, ejection fraction; SV, stroke volume; CO, cardiac output; EDP, end-diastolic pressure. ${ }^{A} P<0.05$ versus control. ${ }^{B} P<0.05$ versus iTAC. Comparisons were made using Student's $t$ test with Bonferroni correction for multiple comparisons.

and desensitization of $\beta A R s$ (Figure $5 \mathrm{~F}$ and data not shown). As expected, PI $3 \mathrm{~K} \gamma_{\text {inact }}$ overexpression significantly displaced the endogenous PI3K enzyme resulting in reduced $\beta$ AR-targeted PI3K activity in iTAC $\gamma_{\text {inact }}$ mice without affecting total $\beta A R K 1$ levels (Supplemental Figure 4). Importantly, the significant downregulation of $\beta A R$ s found in ITAC and CTAC hearts was completely reversed in iTAC $\gamma_{\text {inact }}$ hearts (Figure 5F). Moreover, ISO stimulation of adenylyl cyclase activity was normalized in iTAC $\gamma_{\text {inact }}$ hearts, indicating preserved $\beta A R-G$ protein coupling (data not shown).

Along with these marked abnormalities in $\beta A R$ signaling, histological evaluation of cardiac sections derived from iTAC hearts after only 1 week of pathological stress revealed scattered fibrosis (Figure 6A, middle panels) and marked loss of capillary density (Figure 6A, right panels, and Figure 6B). These abnormalities were also associated with a significant reduction in SERCA2a levels in these hearts (Figure 6C). Remarkably, PI3K $\gamma_{\text {inact }}$ overexpression resulted in the complete prevention of vascular rarefaction (Figure 6, A and B) and normalization of SERCA2a protein levels (Figure 6C) without significantly affecting the level of mild fibrosis (Figure 6A). Taken together, these data indicate that cardiac $\beta$ AR dysfunction is an early hypertrophy-independent pathological event in response to intense but intermittent pressure overload. Importantly, preventing the $\beta A R K 1$ recruitment of PI3K to the plasma membrane is associated with reversal of the $\beta A R$ defect and the development of multiple pathological sequelae in response to pressure overload.

Overexpression of PI3K $\gamma_{\text {inact }}$ rescues early functional abnormalities in iTAC hearts. To determine the functional consequences of the early $\beta A R$ defects and their normalization through overexpression of PI $3 \mathrm{~K} \gamma_{\text {inact, }}$ we measured hemodynamic indices of myocardial systolic and diastolic performance in intact mice from the different groups by in vivo cardiac pressurevolume loop analysis (30) (Table 2). While the hearts of swimming mice exhibited normal diastolic and systolic properties and were indistinguishable from the hearts of control mice (Table 2 and Figure $7, A$ and $B$ ), hearts exposed to either intermittent or chronic pressure overload exhibited a steeper slope of the end-systolic pressurevolume relationship (end-systolic elastance [Ees]; Figure 7, C and E, and Table 2), indicating a hypercontractile state as we and others have previously described $(30,31)$. Diastolic performance was also markedly abnormal in iTAC and cTAC hearts as indicated by significantly delayed relaxation, markedly elevated diastolic pressure, and a steeper end-diastolic pressure-volume relationship (EDPVR) (Table 2). Furthermore, only WT hearts exposed to pathological stress for 1 week (iTAC and cTAC) displayed a blunted contractile response to $\beta$-agonist stimulation in vivo (Figure $7 \mathrm{~F}$ ), consistent with our biochemical findings of abnormal $\beta$ AR signaling. While not affecting $d P / d t_{\max }$ and $d P / d t_{\min }$ (Table 2), PI3K $\gamma_{\text {inact }}$ overexpression completely preserved diastolic function, reduced basal cardiac hypercontractility, and restored the contractile response to $\beta$-agonist stimulation in the iTAC $\gamma_{\text {inact }}$ mice (Figure 7F and Table 2).

Beta blocker treatment preserves $\beta A R$ levels and coupling in response to intermittent pressure overload but has no effect on vascular rarefaction. To further investigate the mechanism by which PI $3 \mathrm{~K} \gamma \gamma_{\text {inact }}$ overexpression exerts its beneficial action, and to compare this novel molecular strategy with beta blocker treatment, we tested the effects of metoprolol on the maladaptive phenotype induced by iTAC (iTAC $\mathrm{C}_{\text {meto }}$ experimental design III; Figure 8A and Supplemental Figure 1D). In this section of the study, we included a new group of controls in which the iTAC suture was placed but never pulled (referred to as the intermittent sham-operated group). Similar to PI $3 \mathrm{~K} \gamma_{\text {inact }}$ overexpression, metoprolol treatment in mice exposed to intermittent pressure overload preserved $\beta$ AR levels (Figure 8B) and their ability to couple to $G$ proteins, as shown by normal in vitro cAMP generation from cardiac membranes stimulated with ISO (Figure 8C). Interestingly, elevated total cardiac cAMP levels were found only in WT iTAC mice, but not in iTAC mice with either PI $3 \mathrm{~K} \gamma_{\text {inact }}$ overexpression or metoprolol treatment (Figure 8D). These results are consistent with recent evidence showing that PI $3 \mathrm{~K} \gamma$ is a member of a multiprotein complex that includes phosphodiesterase $3 \mathrm{~B}$ and therefore could modulate total cAMP levels via a kinase-independent mechanism (32). 
A
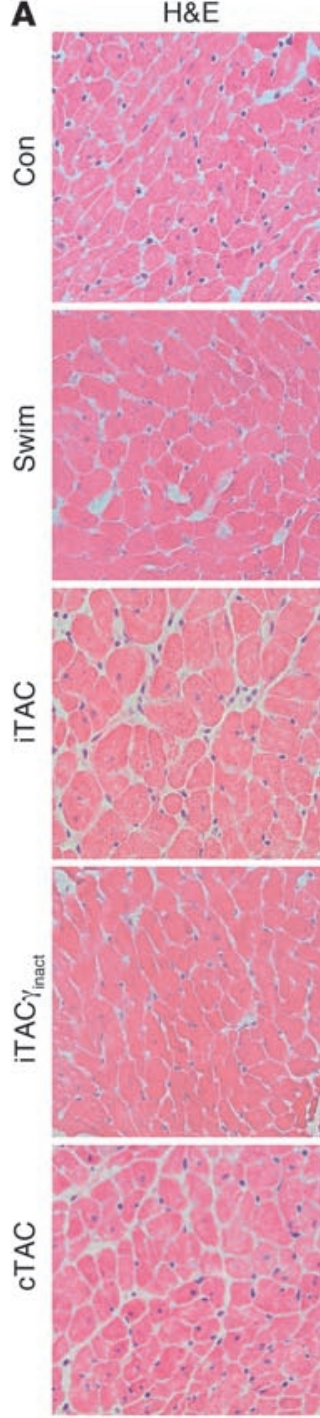
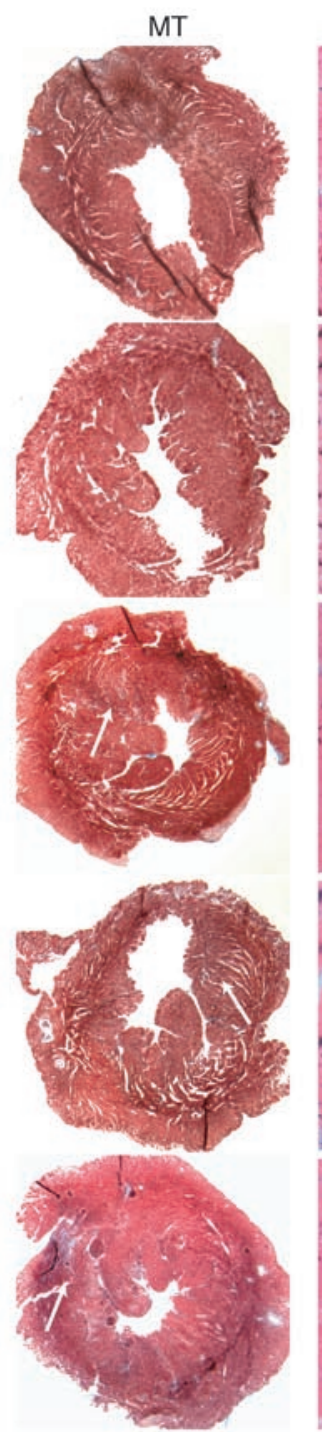

$\mathrm{CD}$
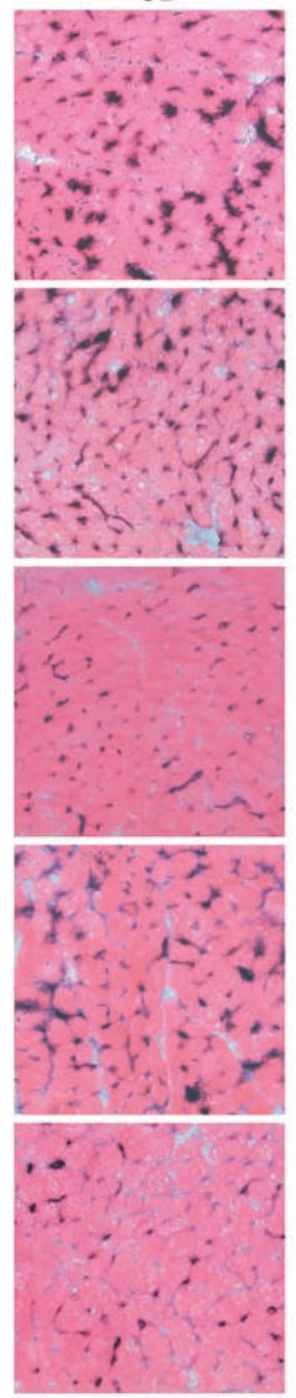

B
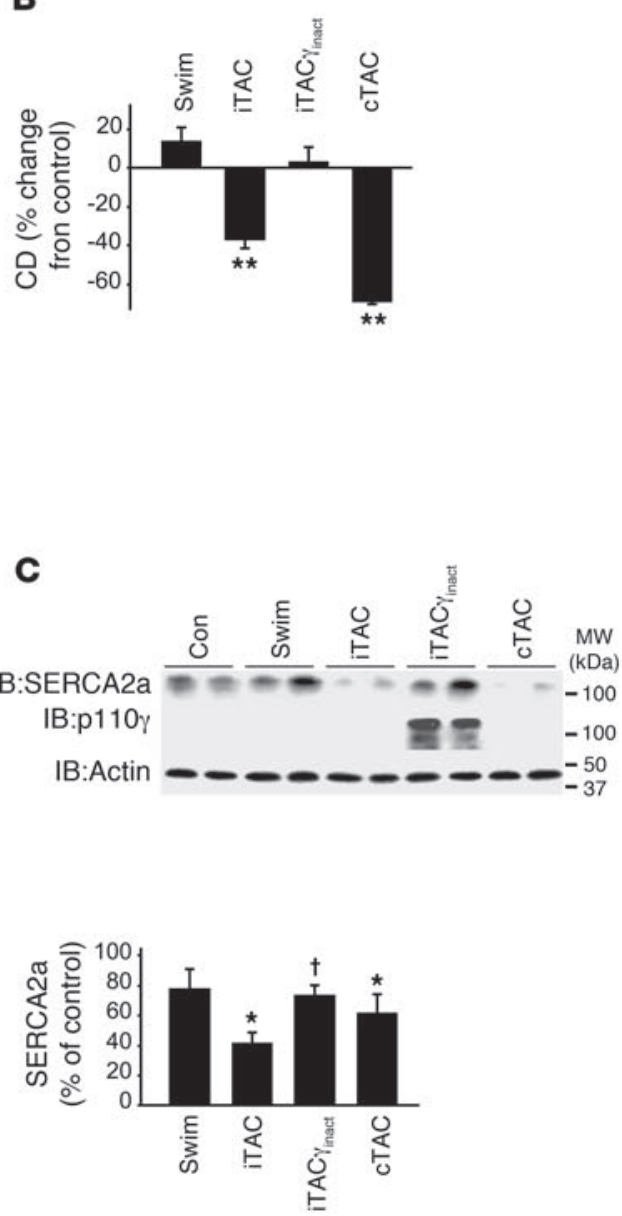

Figure 6

Preservation of $\beta$ AR signaling through targeted PI3K inhibition is associated with preservation of capillary density and SERCA2a levels. (A) Representative staining of cardiac sections with H\&E (magnification, $\times 400$ ), MT (magnification, $\times 20$ ), and endothelial alkaline phosphatase (to determine capillary density; magnification, $\times 400$ ) after 1 week of training. (B) Quantification of capillary density in cardiac sections stained for endothelial alkaline phosphatase expressed as percent reduction from control ( $n=4-6$ hearts per group). ${ }^{* *} P<0.01$ versus control, swimming, and iTAC $\gamma_{\text {inact }}$; Student's $t$ test with Bonferroni correction. (C) Immunoblotting analysis of SERCA2a, actin, and PI3K $\gamma_{\text {inact }}$ protein levels in hearts from experimental design II. Densitometric quantification of SERCA2a levels is shown in the bottom panel $\left(n=6-10\right.$ per group). ${ }^{*} P<0.05$ versus control, ${ }^{\dagger} P<0.05$ versus iTAC, ANOVA with Neuman-Keuls correction.

We next assessed the role of these treatments on apoptotic cell death, performing TUNEL staining on cardiac sections from the different groups. As shown in Figure 8, E and F, PI3K $\gamma_{\text {inact }}$ overexpression as well as metoprolol treatment significantly reduced the rate of apoptotic death of endothelial and cardiac cells compared with WT iTAC mice. Despite these beneficial effects, metoprolol treatment did not prevent vascular rarefaction in iTAC as shown by reduced endothelial alkaline phosphatase staining (Figure 9A) and CD31 immunoreactivity (data not shown). Consistent with the histological findings of vascular rarefaction, cardiac levels of the angiogenic factor angiopoietin 2 were significantly reduced in metoprolol-treated iTAC animals and were similar to untreated iTAC mice. In contrast, we observed a normalization of cardiac angiopoietin
2 levels in iTAC $\gamma_{\text {inact }}$ mice (Figure 9B), in which the overexpression of the catalytically inactive mutant completely rescued the loss in vascular density (Figure 6, A and B). While treatment with the beta blocker metoprolol did not affect the low rate of interstitial and perivascular fibrosis (Figure 9C) or ameliorate the slow rate of isovolumic relaxation observed in WT iTAC mice (Figure 9D), it did significantly reduce the hypercontractile state (Figure 9E) and normalize the slope of the EDPVR relation (Figure 9D) of the intermittently pressure-overloaded hearts. These data support the concept that vascular density and interstitial fibrosis might be involved in the early diastolic dysfunction of pressure-overloaded hearts and progression toward heart failure (33). Moreover, our data indicate that a strategy of displacing PI3K from $\beta$ ARK1 recapitulates many 
A

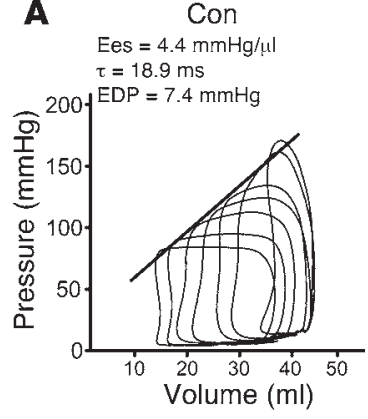

D $\begin{array}{r}\mathrm{iTAC} \gamma_{\text {inact }} \\ \text { Ees }=4.6 \mathrm{mmHg} / \mathrm{\mu l}\end{array}$

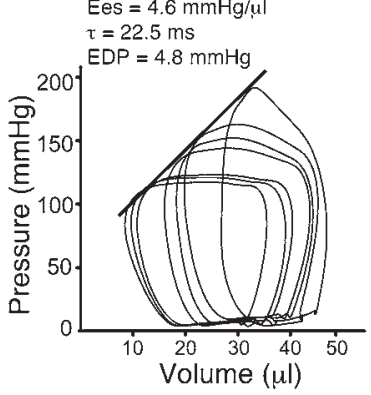

B

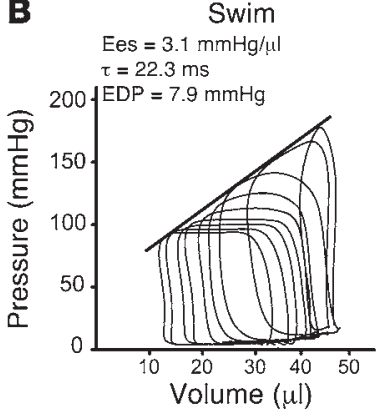

E

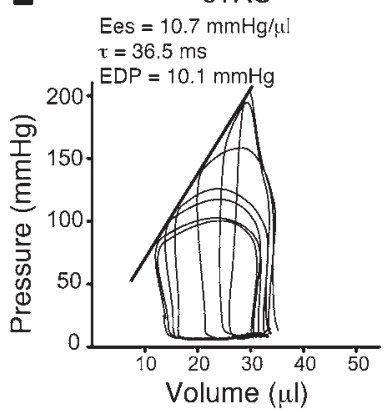

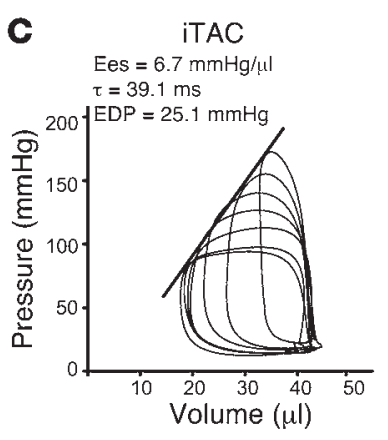

$\mathbf{F}$

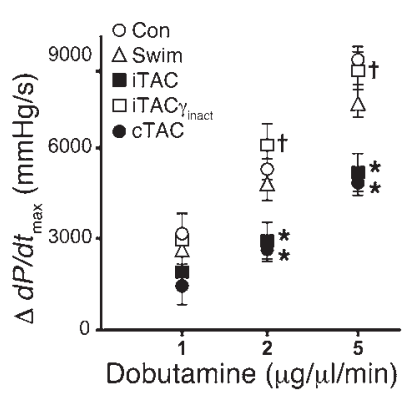

\section{Figure 7}

Targeted PI3K inhibition and normalization of $\beta A R$ signaling ameliorates diastolic dysfunction in hearts exposed to intermittent pressure overloads. (A-E) Representative pressure-volume loops obtained by gently pulling a suture placed around the transverse aorta in control (A), swimming (B), iTAC (C), iTAC $\gamma_{\text {inact }}(\mathbf{D})$, and CTAC (E) mice after 1 week (experimental design II). (F) Change in $d P / d t_{\text {max }}$ in control $(n=11)$, swimming $(n=9)$, iTAC $(n=9)$, iTAC $\gamma_{\text {inact }}$ $(n=9)$, and cTAC $(n=15)$ groups following incremental doses ( 3 minutes each) of dobutamine $(1,2$, and $5 \mu \mathrm{g} / \mu \mathrm{l} / \mathrm{min}) .{ }^{*} P<0.05$ versus control; Student's $t$ test with Bonferroni correction; ${ }^{\dagger} P<0.05$ versus iTAC. of the beneficial effects of beta blockers and exhibits additional protective properties, possibly mediated through novel $G$ proteinindependent signaling pathways $(34,35)$.

The integration of neurohumoral and biomechanical stress triggers early $\beta A R$ dysfunction in hearts exposed to intermittent pressure overload. Our results indicate that the maladaptive hypertrophy-independent phenotype induced by intermittent pressure overload is associated with early $\beta A R$ dysfunction and that preservation of $\beta A R$ signaling can inhibit the development of many of the pathological features. However, the nature of the stress responsible for the induction of early $\beta A R$ abnormalities in pressure-overloaded hearts remains unclear. To test whether different levels of catecholamines during the acute phases of stress might explain the different phenotype observed upon swimming or intermittent pressure overload, we measured plasma levels of epinephrine and norepinephrine in the hearts of WT swimming, iTAC, and iTAC $\gamma_{\text {inact }}$ mice at the termination of the training sessions and after 12 hours of rest, with or without pretreatment with metoprolol (Figure 10) (13, 26, 27). Interestingly, in animals from all the groups terminated immediately after 90 minutes of stress we observed an equal, significant increase in the levels of epinephrine and norepinephrine (Figure 10, A and B) that was similarly reversed by 12 hours of rest. As expected, the treatment of the animals with the beta blocker metoprolol did not affect the neurohumoral activation in these stressed animals (Figure 10, A and B).

Remarkably, despite the equal levels of epinephrine and norepinephrine among the groups (Figure 10, A and B), only iTAC hearts displayed defective $\beta A R / G$ protein coupling after 90 minutes of intermittent pressure overload, consistent with receptor desensitization. These abnormalities were similarly prevented by metoprolol treatment and PI $3 \mathrm{~K} \gamma_{\text {inact }}$ overexpression and were initially reversed by 12 hours of rest (Figure 10C). In the same hearts, we did not detect any changes in plasma membrane $\beta A R$ levels (Figure 10D).

These results demonstrate that swimming and iTAC protocols induce intense, but equal, transient activation of the sympathetic nervous system. However, the marked increase in neurohumoral stimu- lation in vivo by itself is not sufficient to induce the significant early $\beta A R$ desensitization observed with a brief period of pressure overload. While it is not known whether mechanical stress by itself might affect BAR signaling as previously shown for angiotensin II receptors (36), we hypothesize that the network integration of neurohumoral and biomechanical stress might be required to promote $\beta A R$ dysfunction in hearts exposed to intermittent pressure overload.

\section{Discussion}

In this study we address a long-standing fundamental question: Is it the nature of the stress, or the chronicity of the stress, that promotes a pathological cardiac phenotype? Here we demonstrate that the duration of cardiac stress was largely responsible for the amount of cardiac hypertrophy and reexpression of fetal genes, while the nature of the overload determined the phenotype, i.e., whether it is physiological or pathological. We show that intermittent pressure overload resulted in the chronic activation of signaling pathways associated with profound histological and cellular abnormalities despite the seemingly physiologic phenotype of mild hypertrophy, preserved global cardiac function, and absence of fetal gene reexpression. Moreover, our data highlight what we believe to be the novel finding that the combination of excessive neurohumoral activation and biomechanical stress triggered early abnormalities in the $\beta$ AR system. Lastly, a strategy that alters the local generation of phosphatidylinositols by PI3K within the activated $\beta A R$ complex can rescue many of the structural and functional abnormalities induced by pressure overload without exerting any effect on the hypertrophic growth of the heart.

The mechanism by which the heart interprets the nature of increased workloads to cause variable amounts of cardiac enlargement and divergent phenotypes remains unclear. Against the longstanding hypothesis that the duration of the stress plays a critical role in this process (1), we propose that the pathological nature of cardiac stress determines the molecular, functional, and structural signatures of the maladaptive phenotype. Here we show that 
A
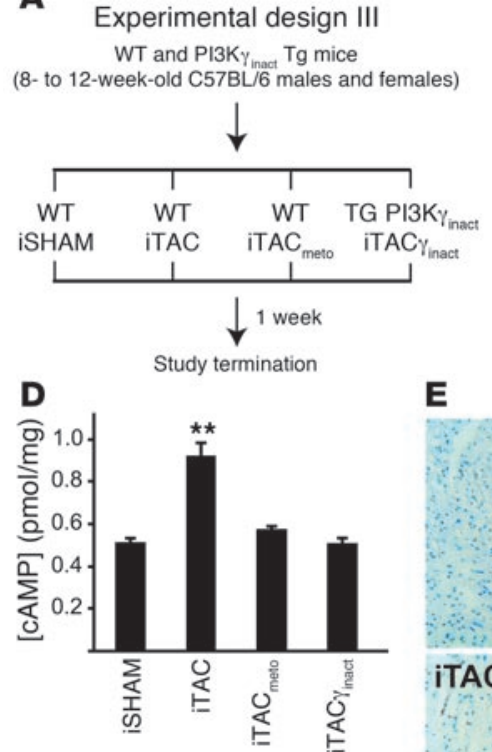

$\mathbf{E}$
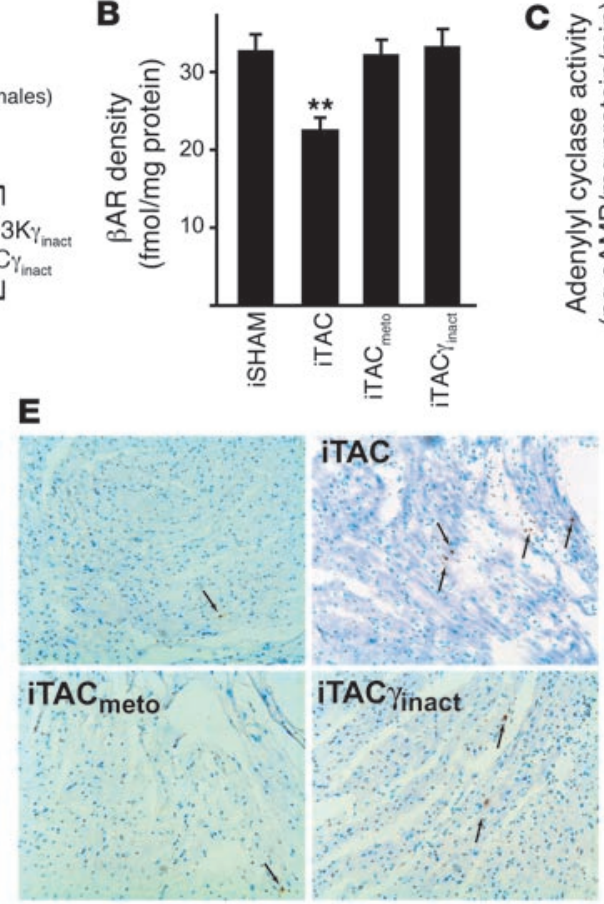

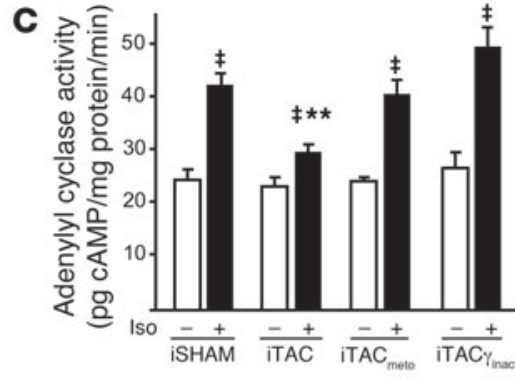

$\mathbf{F}$

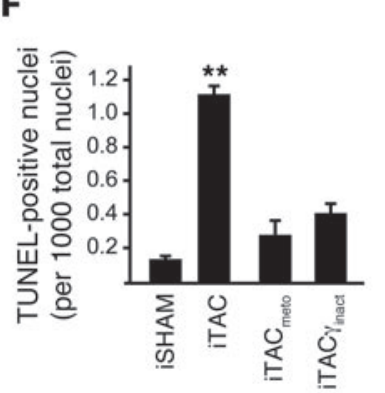

Figure 8

Beta blocker treatment preserves $\beta$ AR levels and coupling in response to intermittent pressure overload. (A) Experimental design III. (B) $\beta$ AR density from the cardiac membrane of hearts from the different groups: intermittent sham-operated (iSHAM) mice, in which the iTAC suture was placed but never pulled $(n=6)$, as well as iTAC $(n=6)$, iTAC meto $(n=6)$, and iTAC $\gamma_{\text {inact }}$ mice $(n=6)$. (C) Adenylyl cyclase activity at basal levels (white bars) and upon ISO stimulation (black bars). ${ }^{\ddagger} P<0.01$ versus respective basal group; ${ }^{* *} P<0.01$ versus intermittent sham-operated ISO; ANOVA with Bonferroni correction. (D) Intracellular concentration of cAMP in cardiac total cell lysates. ${ }^{* *} P<0.01$ versus all groups; ANOVA with Bonferroni correction. (E) Representative TUNEL staining of cardiac sections. Arrows indicate apoptotic cell nuclei. (F) Summary data of multiple independent experiments. ${ }^{* \star} P<0.01$ versus intermittent sham-operated mice.

the amount of cardiac hypertrophy and fetal gene reexpression is proportional to the duration of stress, since mice exposed to the same pathological stress, either intermittently or chronically, significantly differed in the magnitude of the cardiac hypertrophic response and the fetal gene expression profile. In contrast, mice exposed to either physiological or pathological stress for the same duration (swimming and iTAC) displayed a similar lack of fetal gene reinduction. While recent data show that the reprogramming of gene expression can be dissociated from cardiac growth (3), it usually accompanies the maladaptive phenotype $(21,37)$. Our data suggest that while fetal gene reexpression is a valuable marker in established pathological conditions $(21,37)$ and potentially can be used to monitor the efficacy of drug therapy in heart failure (38), its absence does not indicate a physiological adaptation to stress. Indeed, we show that a markedly abnormal structural and functional phenotype can exist without significant changes in the gene expression of critical genes like $\beta \mathrm{MHC}$, atrial natriuretic peptide, or brain natriuretic peptide and imply that a nuclear "pathological switch" can only be induced by stresses that are chronic. Hence the favorable fetal gene expression profile of a mildly hypertrophic heart can coexist with molecular signaling pathways, leading to a markedly abnormal phenotype.

An intricate network of intracellular signaling pathways activated by cardiac stress promotes the hypertrophic response to induce either a pathological or a physiological phenotype (39). Even though cardiac hypertrophy is now recognized as an independent cardiovas- cular risk factor, its specific role is unclear, since inhibition of cardiac growth can be either beneficial (4) or detrimental (8-12) and stimulation of cardiac hypertrophy is not always maladaptive $(15,40)$. While we cannot exclude that the mild hypertrophic response seen after 4 weeks of iTAC may be important to preserve cardiac function in spite of the intermittent increases in afterload, our short-term studies showed that the activation of pathogenic signaling pathways can be dissociated from the induction of cardiac growth since they were activated after only 7 days of intermittent cardiac overload, when no significant cardiac growth was detected. Moreover, similar levels of cardiac growth can be linked to either a pathological or a physiological phenotype, suggesting that hypertrophy itself may not be the critical determinant of cardiac failure as currently believed (3, $6,7)$. In contrast, the molecular signature of the overloaded heart is the early initiator of the beneficial or detrimental fate, and the inhibition of pathogenic signaling pathways can rescue the pathological phenotype without affecting the growth response.

In our current study we showed that $\beta A R$ abnormalities are early cardiac events in response to pathological loads. Consistent with previous work, one of the earliest events in the process leading to $\beta A R$ dysfunction is the increase in $\beta A R K 1$ levels (23). Interestingly, the mechanism for the increase in $\beta A R K 1$ levels and the development of $\beta A R$ dysfunction seems to be disconnected from the induction of cellular hypertrophy, as no cardiac hypertrophy was observed following a week of intermittent pressure overload. Moreover, in contrast to the concept that increased levels of circu- 
A
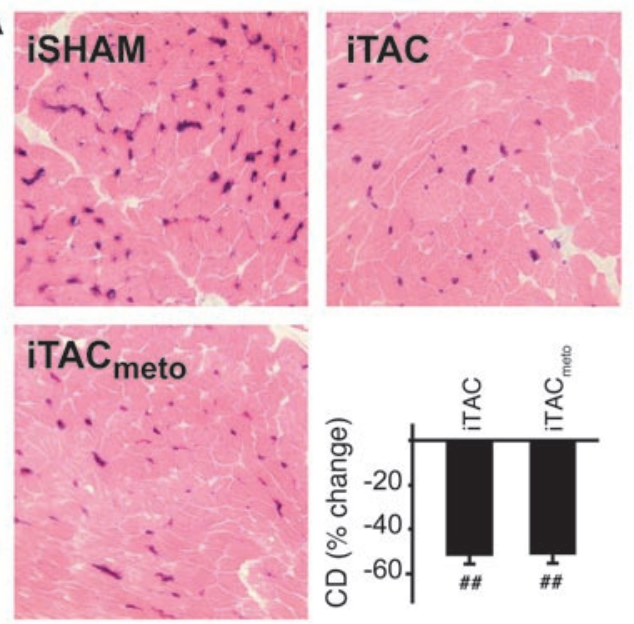
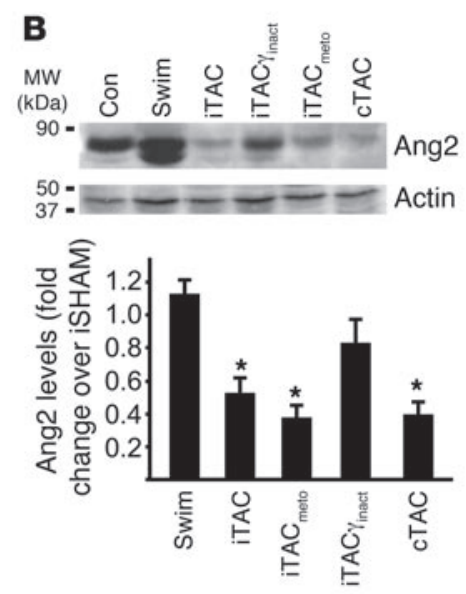

\section{Figure 9}

Effects of metoprolol treatment on vascular density, fibrosis, and in vivo indices of systolic and diastolic function. (A) Representative images of cardiac sections stained for endothelial alkaline phosphatase (magnification, $\times 400$ ) and summary data of different independent experiments. (B) Representative immunoblots of cardiac lysates for angiopoietin 2 (Ang2) and relative densitometric and statistical analysis of multiple independent experiments. ${ }^{*} P<0.05, \# P<0.01$ versus intermittent sham-operated mice. (C) Bar graphs showing percent area of fibrosis in the different groups. ${ }^{\#} P<0.01$ versus all other groups. (D-F) Summary data for $\tau$ Glantz (D), Ees (E), and the slope of the EDPVR (F). ${ }^{*} P<0.05$ versus control; Student's $t$ test with Bonferroni correction.

C

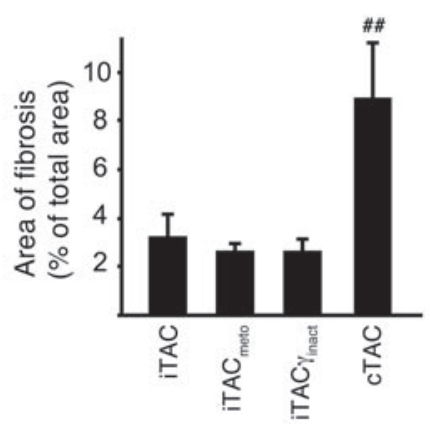

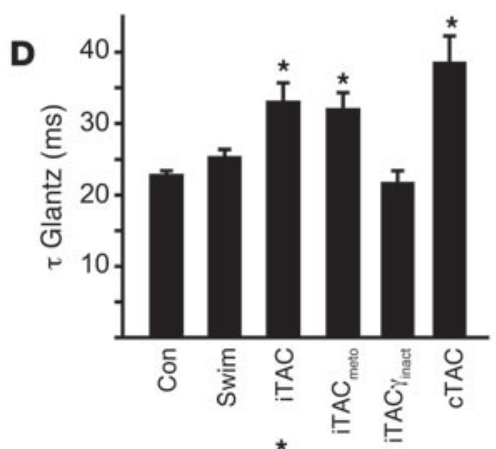

E

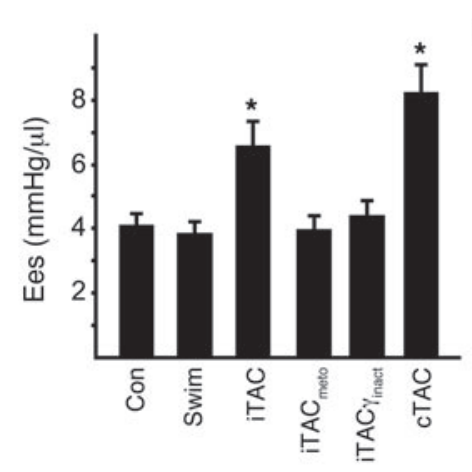

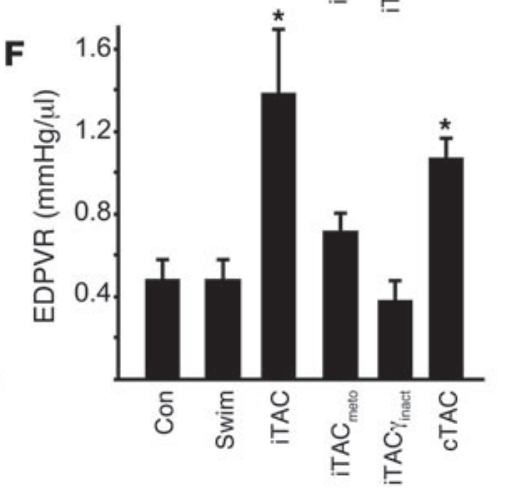

the maladaptive phenotype without modulating cardiac growth. These results are consistent with our recent studies showing that preventing the local generation of phosphatidylinositols by PI3K within the activated receptor complex preserves $\beta A R$ signaling and ameliorates cardiac dysfunction in a variety of animal models of heart failure (13, $26,27)$. While we cannot exclude that early $\beta A R$ hyperfunction might be involved in the development of the maladaptive phenotype, our approach of displacing the PI3K from $\beta A R K 1$ prevented many pathological features induced by pressure overload by normalizing $\beta A R$ signaling in a manner similar to the beta blocker metoprolol. Interestingly, while exerting multiple beneficial effects in response to intermittent pressure overload, metoprolol treatment did not preserve vascular density or the rate of isovolumic relaxation in ITAC mice. While it is well known that beta blockers can increase the rate of isovolumic relaxation in the intact heart (41, 42 ), our data suggest that the mechanism(s) for the loss in vascular density with pressure overload appear to be independent from cat-

lating catecholamines in human heart failure are solely responsible for $\beta A R$ dysfunction, here we showed that physical training and transient pressure overload both produced intense neurohumoral activation, yet only pressure overload resulted in $\beta A R$ desensitization and downregulation. These results strongly suggest that the combination of excessive neurohumoral activation and increased mechanical load is required to trigger abnormalities in the $\beta A R$ system. While it is not known whether $\beta$ ARs might function as sensors of mechanical stress signals as recently shown for angiotensin II type 1 receptor (36), we postulate that the intracellular integration of neurohumoral and biomechanical stimuli promotes the activation of distinct signaling pathways leading to divergent phenotypes. What role the specific $\beta A R$ subtypes and/or the angiotensin II receptor plays in the development of this maladaptive phenotype is not certain and will need to be addressed in future studies.

Our study shows that preservation of $\beta A R$ signaling through a strategy of displacing PI3K from $\beta A R K 1$ rescued many features of echolamine stimulation of $\beta$ ARs since it was not prevented by beta blocker treatment. In addition, these data suggest that disrupting the $\beta A R K 1 / P I 3 K$ complex might exert beneficial effects that are independent of $\beta A R / G$ protein coupling $(13,24,26,27)$ and cAMP levels $(32,43)$, possibly through the activation of protective $G$ protein-independent signaling pathways $(34,35)$.

Classically, G protein-coupled receptors (GPCRs) transduce extracellular signals by coupling to heterotrimeric $G$ proteins, although recent studies have suggested that some aspects of GPCRmediated signaling can occur independent of $G$ protein activation $(34,35)$. The proximal regulators for this $G$ protein-independent signal transduction appear to be GPCR kinases (GRKs, of which $\beta A R K 1$ is a family member) and $\beta$-arrestins. While the sequential action of GRKs and $\beta$-arrestins on the receptor result in the waning of $G$ protein-dependent signals, a process known as GPCR desensitization (24), recent evidence suggests that GRKs and $\beta$-arrestins also perform independent signal transducing functions 
A

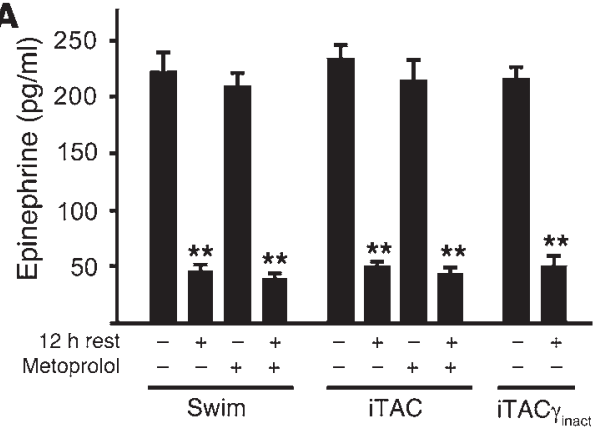

C

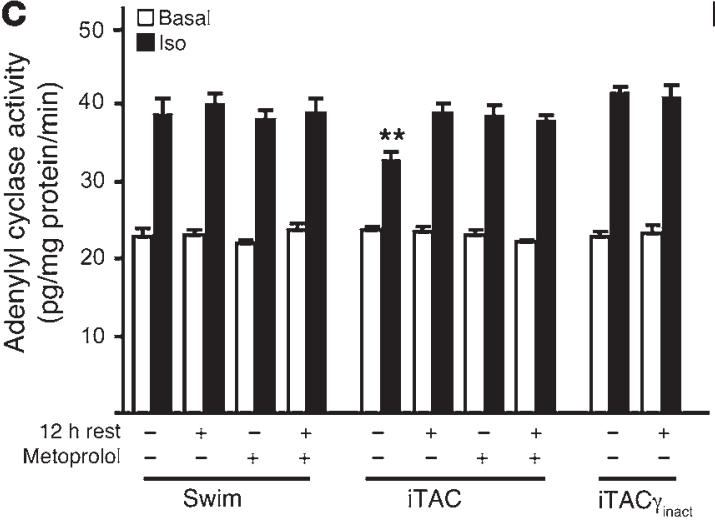

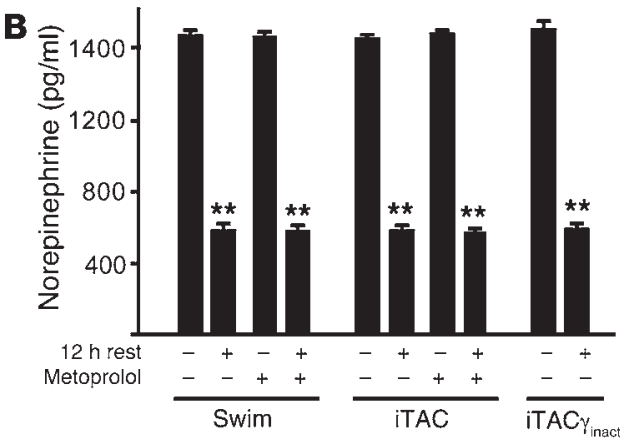

D

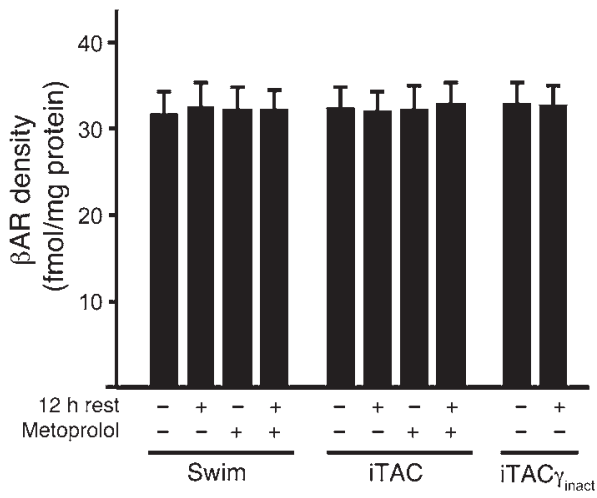

Figure 10

The combination of neurohumoral and biomechanical stress is required to trigger early $\beta A R$ dysfunction in stressed hearts. Plasma levels of catecholamines epinephrine (A) and norepinephrine (B) as well as in vitro membrane generation of cAMP (C) and $\beta A R$ density (D) in cardiac membranes prepared from hearts excised immediately after termination of the training protocol or after 12 hours of rest, in the presence or absence of metoprolol before treatment. ${ }^{* *} P<0.01$ versus any stress $(\mathbf{A}$ and $\mathbf{B})$ or versus swimming ISO and iTAC $\gamma_{\text {inact }}$ ISO $(\mathbf{C})$.

to initiate new $\mathrm{G}$ protein-independent signaling pathways (35). We have previously shown that overexpression of $\mathrm{PI} 3 \mathrm{~K} \gamma_{\text {inact }}$ allows for the efficient recruitment of both $\beta$ ARK 1 and $\beta$-arrestin to agonist-stimulated $\beta$ ARs $(44,45)$. Our present data, which showed a rescue of the vascular rarefaction in the iTAC $\gamma_{\text {inact }}$ mice but not in the iTAC mice treated with beta blockers, suggests that a potential mechanism for this salutary effect could be due to activation of protective $\beta$-arrestin-mediated signaling pathways.

Although previous studies as well as the present one indicate that the PI $3 \mathrm{~K} \gamma$ isoform is selectively activated by pathological pressure overload, it has been shown in PI3K $\gamma$ knockout mice that the absence of PI3K $\gamma$ alone is not sufficient to preserve $\beta$ AR signaling following chronic catecholamine stimulation (13) or cardiac function in response to pressure overload (32). These results are consistent with our hypothesis that it is not the absence of PI3K $\gamma$, but the displacement of all catalytically active PI3K isoforms from the receptor complex, that is critical to prevent $\beta$ AR dysfunction, since the $\beta A R K 1$-interacting domain is conserved among all class I $\mathrm{PI} 3 \mathrm{Ks}$ and therefore other PI3K active isoforms can be recruited to the receptor complex.

Interestingly, and consistent with our results, intermittent increases in arterial blood pressure that occur in patients with obstructive sleep apnea (46) are associated with a gradual deterioration of LV diastolic function (47) and an increased risk of cardiovascular disease, even when adjusted for multiple coexisting risk factors (48).

In conclusion, we showed that cardiac hypertrophy is a timedependent and general response to cardiac stress irrespective of the underlying molecular, structural, and functional phenotype. Intermittent pressure overload induced an early hypertrophy-independent transition of cardiomyocytes into a pathological phenotype that was markedly ameliorated through a strategy that prevented the recruitment of endogenous PI3K to ligand-activated GPCRs.

\section{Methods}

Experimental animals. WT C57BL/6 female mice were used for the 4-week study. WT and transgenic mice of both genders, all inbred on a C57BL/6 background, were used for the 1-week study. Transgenic mice overexpressing a catalytically inactive form of PI3K $\gamma$ (iTAC $\gamma_{\text {inact }}$ mice) have been previously described $(13,26)$. All animals were handled according to the approved protocols and animal welfare regulations of the Institutional Review Board at Duke University Medical Center.

Exercise models of physiological hypertrophy. Physiological hypertrophy was induced in mice as previously described by swimming (15) for 90 minutes twice a day or by voluntary running in wheel-equipped cages (16).

cTAC and iTAC models of pressure overload. Chronic pressure overload in the mouse was induced by cTAC as previously described (17), except that the suture was placed between the left carotid and the left axillary arteries. A model of intermittent pressure overload was established in the mouse by modifying the cTAC procedure to produce iTAC. Briefly, a suture was placed around the aorta between the left carotid and the left axillary arteries, which was then tunneled through the back of the mouse and exteriorized (Supplemental Figure 1A). TAC was obtained by pulling the suture in conscious mice for 90 minutes twice a day. The duration of the intermittent hemodynamic stress was chosen to mimic the duration of the swim- 
ming exercise. At termination of the study, the efficacy of the pressure overload was tested in all animals by measuring the arterial pressures in the right carotid artery (proximal to the suture) and the left axillary artery (distal to the suture) following intermittent constriction (Figure 1B). For iTAC mice, 3 conditions were required for subsequent inclusion in the study: (a) the absence of a basal pressure gradient between proximal and distal artery; (b) an increase of at least $40 \mathrm{mmHg}$ in the systolic pressure of the proximal artery during the intermittent constriction, with or without decrease in the systolic pressure of the distal artery; and (c) the regression of the pressure overload after release of the constricting suture. Three different groups of controls were used in this study: sedentary WT animals (controls), cTAC controls in which the suture was passed around the aorta but not ligated (sham-operated), and iTAC controls in which the suture was passed around the aorta and exteriorized to the back of the mouse but never pulled (intermittent sham-operated).

Administration of beta blocker metoprolol. Beta blocker metoprolol was administered in drinking water $(350 \mathrm{mg} / \mathrm{kg} \mathrm{BW} / \mathrm{d})$ as previously shown (49) beginning 7 days before the iTAC surgery until the study termination (experimental design III, Supplemental Figure 1D).

Transthoracic echocardiography. Serial echocardiography was performed on conscious mice from all groups with an HDI 5000 echocardiograph (Philips) and a Vevo 770 high-resolution imaging system (VisualSonics) after 4 weeks as previously described (26).

Gene expression analysis. At study termination, hearts were snap-frozen in liquid nitrogen and stored at $-80^{\circ} \mathrm{C}$. RNA samples were prepared from powdered tissue and extracted with the use of Tripure (Roche Diagnostics Corp.) according to the manufacturer's instructions. mRNA expression of selected genes was carried out by real-time quantitative reverse transcription-PCR with a ABI 6700 machine $(18,50,51)$. All reactions included an actin internal standard.

Histological studies. Freshly harvested cardiac samples were placed in sucrose/phosphate-buffered saline solution at $4^{\circ} \mathrm{C}$ for $2-4$ hours, placed in cross-section in OCT (Miles Pharmaceuticals), and snap-frozen in liquid nitrogen. Slides from frozen sections were subsequently fixed in $4 \%$ paraformaldehyde and stained with H\&E and MT. Capillary density in the heart was measured by endogenous endothelial alkaline phosphatase staining on frozen sections as previously described (52). Capillary density was quantified by examining 6-10 random high-power fields (magnification, $\times 400$ ) on an inverted light microscope. Photographs were taken using an Optometrics analog camera and Adobe Premier version 5.1, and these images were analyzed using an NIH Image analysis system.

Determination of plasma catecholamine levels. Plasma levels of catecholamines epinephrine and norepinephrine were measured using the catecholamine assay kit Bi-CAT EIA (17-EA613-192; ALPCO Diagnostics) according to the manufacturer's instructions.

Determination of total intracellular cAMP levels. Total cAMP levels were measured using the AMP-[H3] biotrak assay (TRK432; Amersham Biosciences) according to the manufacturer's instructions.

Primary culture of cardiac myocytes and cell contractility. Cardiac myocytes were isolated from mouse hearts as described previously (53) and used for contractility studies. Cells of similar lengths were selected, and single-cell contractions were measured in rod-shaped cells by video edge detection (Crescent Electronics). Recordings were made upon electric field stimulation under basal conditions and following administration of ISO $1 \mu \mathrm{M}$ as previously described (27). The extent of twitch shortening (percent CS) was calculated as follows: (maximum length - minimum length) $\times 100 /$ maximum length. In each animal, 10-15 cells were analyzed.

Membrane fractionation, $\beta A R$ radioligand binding, and adenylyl cyclase activity. Membrane and cytosolic fractions from LVs flash-frozen in liquid $\mathrm{N}_{2}$ were prepared as described previously (4). Receptor binding with $20 \mu \mathrm{g}$ of proteins from the membrane fraction was performed as described previously (4) using $\beta$ AR ligand [ $\left.{ }^{125} \mathrm{I}\right]$ cyanopindolol $(250 \mathrm{pM})$. All assays were performed in triplicate, and receptor density (fmol) was normalized to milligrams of membrane protein. Adenylyl cyclase assays were performed as described previously (4), using $20 \mu \mathrm{g}$ of the membrane fraction. Generated cAMP was quantified using a liquid scintillation counter (MINAXI 4000; Packard Instrument Co.).

Immunoprecipitation and immunoblotting. Immunoprecipitation and immunoblotting were performed as described previously (13). Detection was carried out using ECL (Amersham Biosciences), and bands were quantified using Bio-Rad Flouro-S Multimage software version 1.0.

PI3K activity. PI3K assays were carried out by immunoprecipitation of the PI3K $\alpha$ and $-\gamma$ isoforms from the cytosolic fraction as described previously (54). $\beta A R K 1$-associated PI3K activity was measured after immunoprecipitation of $400 \mu \mathrm{g}$ of proteins from the membrane fraction with a polyclonal antibody directed against $\beta$ ARK1 (Santa Cruz Biotechnology Inc.). Lipids were extracted with chloroform/methanol (1:1 ratio), and the organic phase was spotted on TLC plates and resolved chromatographically with $2 \mathrm{~N}$ glacial acetic acid/1-propanol (35:65 ratio). Dried plates were exposed, and autoradiographic signals were quantified using Bio-Rad Flouro-S Multimage software version 1.0.

Mitogen-activated protein kinase activity. Mitogen-activated protein kinase activities were assessed from LV cytosolic extracts as the capacity of immunoprecipitated ERK2-p42/ERK1-p44, JNK1, and p38 (Santa Cruz Biotechnology Inc.) to phosphorylate in vitro substrates (myelin basic protein and GST-cJun) as previously described (26). Total protein levels for each kinase were assessed by immunoblotting.

Pressure-volume loops analysis in anesthetized mice. Mice were anesthetized with ketamine $(100 \mathrm{mg} / \mathrm{Kg})$ and xylazine $(2.5 \mathrm{mg} / \mathrm{kg})$ and connected to a rodent ventilator after endotracheal intubation. The surgical suture was cut in cTAC animals. After bilateral vagotomy, a new suture was placed around the transverse aorta of all animals for the transient augmentation of afterload. Cardiac catheterization was performed using a 1.4 French $(0.46 \mathrm{~mm})$ conductance catheter (Millar Instruments Inc.) inserted retrograde through the right carotid artery into the LV and a polyethylene-50 catheter placed into the right external jugular vein for dobutamine infusion. Steady-state pressure and volume measurements were recorded at baseline and after 3 -minute dobutamine infusions $(1,2$, and $5 \mu \mathrm{g} / \mathrm{kg} / \mathrm{min})$. Pressure and volume measurements were also acquired during the increase in the afterload generated by gently pulling on the suture to transiently constrict the aorta. Data were recorded digitally at $1,000 \mathrm{~Hz}$ and analyzed with PVAN analysis software (version 3.3; Millar Instruments Inc.).

Statistics. Data are expressed as mean \pm SEM. Multigroup comparisons were performed using 2-tailed Student's $t$ test or 1-way ANOVA with post hoc correction for multiple comparisons. For all analyses, $P<0.05$ was considered significant.

\section{Acknowledgments}

This work was supported by NIH grants to H.A. Rockman (PO1 HL 75443) and to O. Smithies (HL 49277 and HL 71266). We gratefully thank Brian Annex for his thoughtful suggestions and Kristine Hesser Porter for her excellent technical work.

Received for publication April 18, 2005, and accepted in revised form March 21, 2006.

Address correspondence to: Howard A. Rockman, Departments of Medicine, Cell Biology and Molecular Genetics, DUMC 3104, Room 226 CARL Building, Durham, North Carolina 27710, USA. Phone: (919) 668-2520; Fax: (919) 668-2524; E-mail: h.rockman@duke.edu. 
1. Meerson, F.Z. 1962. Compensatory hyperfunction of the heart and cardiac insufficiency. Circ. Res. 10:250-258.

2. Levy, D., Garrison, R.J., Savage, D.D., Kannel, W.B., and Castelli, W.P. 1990. Prognostic implications of echocardiographically determined left ventricular mass in the Framingham Heart Study. N. Engl. J. Med. 322:1561-1566.

3. Frey, N., Katus, H.A., Olson, E.N., and Hill, J.A. 2004. Hypertrophy of the heart: a new therapeutic target? Circulation. 109:1580-1589.

4. Esposito, G., et al. 2002. Genetic alterations that inhibit in vivo pressure-overload hypertrophy prevent cardiac dysfunction despite increased wall stress. Circulation. 105:85-92.

5. Yusuf, S., et al. 2000. Effects of an angiotensin-converting-enzyme inhibitor, ramipril, on cardiovascular events in high-risk patients. The Heart Outcomes Prevention Evaluation Study Investigators. N. Engl. J. Med. 342:145-153.

6. Devereux, R.B., et al. 2004. Prognostic significance of left ventricular mass change during treatment of hypertension. JAMA. 292:2350-2356.

7. Gardin, J.M., and Lauer, M.S. 2004. Left ventricular hypertrophy: the next treatable, silent killer? JAMA 292:2396-2398.

8. Badorff, C., et al. 2002. Fas receptor signaling inhibits glycogen synthase kinase $3 \beta$ and induces cardiac hypertrophy following pressure overload. J. Clin. Invest. 109:373-381. doi:10.1172/JCI200213779.

9. Brancaccio, M., et al. 2003. Melusin, a muscle-specific integrin beta1-interacting protein, is required to prevent cardiac failure in response to chronic pressure overload. Nat. Med. 9:68-75.

10. Hirota, H., et al. 1999. Loss of a gp130 cardiac muscle cell survival pathway is a critical event in the onset of heart failure during biomechanical stress. Cell. 97:189-198.

11. Meguro, T., et al. 1999. Cyclosporine attenuates pressure-overload hypertrophy in mice while enhancing susceptibility to decompensation and heart failure. Circ. Res. 84:735-740.

12. Rogers, J.H., et al. 1999. RGS4 causes increased mortality and reduced cardiac hypertrophy in response to pressure overload. J. Clin. Invest. 104:567-576.

13. Nienaber, J.J., et al. 2003. Inhibition of receptor-localized PI3K preserves cardiac $\beta$-adrenergic receptor function and ameliorates pressure overload heart failure. J. Clin. Invest. 112:1067-1079. doi:10.1172/JCI200318213.

14. Tachibana, H., Naga Prasad, S.V., Lefkowitz, R.J., Koch, W.J., and Rockman, H.A. 2005. Level of betaadrenergic receptor kinase 1 inhibition determines degree of cardiac dysfunction after chronic pressure overload-induced heart failure. Circulation. 111:591-597.

15. McMullen, J.R., et al. 2003. Phosphoinositide 3-kinase(p110alpha) plays a critical role for the induction of physiological, but not pathological, cardiac hypertrophy. Proc. Natl. Acad. Sci. U. S. A. 100:12355-12360.

16. Akimoto, T., Ribar, T.J., Williams, R.S., and Yan, Z. 2004. Skeletal muscle adaptation in response to voluntary running in $\mathrm{Ca} 2+/$ calmodulin-dependent protein kinase IV-deficient mice. Am. J. Physiol. Cell Physiol. 287:C1311-C1319.

17. Rockman, H.A., et al. 1991. Segregation of atrialspecific and inducible expression of an atrial natriuretic factor transgene in an in vivo murine model of cardiac hypertrophy. Proc. Natl. Acad. Sci. U. S. A. 88:8277-8281.

18. Rapacciuolo, A., et al. 2001. Important role of endogenous norepinephrine and epinephrine in the development of in vivo pressure-overload car- diac hypertrophy. J. Am. Coll. Cardiol. 38:876-882.

19. Christensen, N.J., and Galbo, H. 1983. Sympathetic nervous activity during exercise. Annu. Rev. Physiol. 45:139-153.

20. Liang, C., Tuttle, R.R., Hood, W.B., Jr., and Gavras, H. 1979. Conditioning effects of chronic infusions of dobutamine. Comparison with exercise training. J. Clin. Invest. 64:613-619.

21. Lowes, B.D., et al. 1997. Changes in gene expression in the intact human heart. Downregulation of $\alpha$-myosin heavy chain in hypertrophied, failing ventricular myocardium. J. Clin. Invest. 100:2315-2324.

22. Nakao, K., Minobe, W., Roden, R., Bristow, M.R., and Leinwand, L.A. 1997. Myosin heavy chain gene expression in human heart failure. J. Clin. Invest. 100:2362-2370.

23. Cho, M.C., et al. 1999. Defective beta-adrenergic receptor signaling precedes the development of dilated cardiomyopathy in transgenic mice with calsequestrin overexpression. J. Biol. Chem. 274:22251-22256.

24. Rockman, H.A., Koch, W.J., and Lefkowitz, R.J. 2002. Seven-transmembrane-spanning receptors and heart function. Nature. 415:206-212.

25. Choi, D.J., Koch, W.J., Hunter, J.J., and Rockman, H.A. 1997. Mechanism of beta-adrenergic receptor desensitization in cardiac hypertrophy is increased beta-adrenergic receptor kinase. J. Biol. Chem. 272:17223-17229.

26. Perrino, C., Naga Prasad, S.V., Patel, M., Wolf, M.J., and Rockman, H.A. 2005. Targeted inhibition of beta-adrenergic receptor kinase-1-associated phosphoinositide- 3 kinase activity preserves beta-adrenergic receptor signaling and prolongs survival in heart failure induced by calsequestrin overexpression. J. Am. Coll. Cardiol. 45:1862-1870.

27. Perrino, C., et al. 2005. Restoration of beta-adrenergic receptor signaling and contractile function in heart failure by disruption of the betaARK1/ phosphoinositide 3-kinase complex. Circulation. 111:2579-2587.

28. Sadoshima, J., et al. 2002. The MEKK1-JNK pathway plays a protective role in pressure overload but does not mediate cardiac hypertrophy. J. Clin. Invest. 110:271-279. doi:10.1172/JCI200214938.

29. Zhang, D., et al. 2000. TAK1 is activated in the myocardium after pressure overload and is sufficient to provoke heart failure in transgenic mice. Nat. Med. 6:556-563.

30. Takaoka, H., Esposito, G., Mao, L., Suga, H., and Rockman, H.A. 2002. Heart size-independent analysis of myocardial function in murine pressure overload hypertrophy. Am. J. Physiol. Heart Circ. Physiol. 282:H2190-H2197.

31. Sasayama, S., Franklin, D., and Ross, J., Jr. 1977. Hyperfunction with normal inotropic state of the hypertrophied left ventricle. Am. J. Physiol. 232:H418-H425.

32. Patrucco, E., et al. 2004. PI3Kgamma modulates the cardiac response to chronic pressure overload by distinct kinase-dependent and -independent effects. Cell. 118:375-387.

33. Shiojima, I., et al. 2005. Disruption of coordinated cardiac hypertrophy and angiogenesis contributes to the transition to heart failure. J. Clin. Invest. 115:2108-2118. doi:10.1172/JCI24682.

34. Lefkowitz, R.J., and Shenoy, S.K. 2005. Transduction of receptor signals by beta-arrestins. Science. 308:512-517.

35. Rajagopal, K., Lefkowitz, R.J., and Rockman, H.A. 2005. When 7 transmembrane receptors are not G protein-coupled receptors. J. Clin. Invest. 115:2971-2974. doi:10.1172/JCI26950.

36. Zou, Y., et al. 2004. Mechanical stress activates angiotensin II type 1 receptor without the involve- ment of angiotensin II. Nat. Cell Biol. 6:499-506

37. Miyata, S., Minobe, W., Bristow, M.R., and Leinwand, L.A. 2000. Myosin heavy chain isoform expression in the failing and nonfailing human heart. Circ. Res. 86:386-390.

38. Lowes, B.D., et al. 2002. Myocardial gene expression in dilated cardiomyopathy treated with beta-blocking agents. N. Engl. J. Med. 346:1357-1365.

39. Dorn, G.W., II, and Force, T. 2005. Protein kinase cascades in the regulation of cardiac hypertrophy. J. Clin. Invest. 115:527-537. doi:10.1172/ JCI200524178.

40. Bueno, O.F., et al. 2000. The MEK1-ERK1/2 signaling pathway promotes compensated cardiac hypertrophy in transgenic mice. EMBO J. 19:6341-6350.

41. Frederiksen, J.W., Weiss, J.L., and Weisfeldt, M.L. 1978. Time constant of isovolumic pressure fall: determinants in the working left ventricle. Am. J. Physiol. 235:H701-H706.

42. Gaasch, W.H., Blaustein, A.S., Andrias, C.W., Donahue, R.P., and Avitall, B. 1980. Myocardial relaxation. II. Hemodynamic determinants of rate of left ventricular isovolumic pressure decline. Am. J. Physiol. 239:H1-H6.

43. Crackower, M.A., et al. 2002. Regulation of myocardial contractility and cell size by distinct PI3KPTEN signaling pathways. Cell. 110:737-749.

44. Naga Prasad, S.V., Jayatilleke, A., Madamanchi, A., and Rockman, H.A. 2005. Protein kinase activity of phosphoinositide 3-kinase regulates beta-adrenergic receptor endocytosis. Nat. Cell Biol. 7:785-796.

45. Naga Prasad, S.V., et al. 2002. Phosphoinositide 3-kinase regulates beta2-adrenergic receptor endocytosis by AP-2 recruitment to the receptor/betaarrestin complex. J. Cell Biol. 158:563-575.

46. Somers, V.K., Dyken, M.E., Clary, M.P., and Abboud, F.M. 1995. Sympathetic neural mechanisms in obstructive sleep apnea. J. Clin. Invest. 96:1897-1904.

47. Kraiczi, H., Caidahl, K., Samuelsson, A., Peker, Y., and Hedner, J. 2001. Impairment of vascular endothelial function and left ventricular filling: association with the severity of apnea-induced hypoxemia during sleep. Chest. 119:1085-1091.

48. Shahar, E., et al. 2001. Sleep-disordered breathing and cardiovascular disease: cross-sectional results of the Sleep Heart Health Study. Am. J. Respir. Crit. Care Med. 163:19-25.

49. Harding, V.B., Jones, L.R., Lefkowitz, R.J., Koch, W.J., and Rockman, H.A. 2001. Cardiac beta ARK1 inhibition prolongs survival and augments beta blocker therapy in a mouse model of severe heart failure. Proc. Natl. Acad. Sci. U. S. A. 98:5809-5814.

50. Ellmers, L.J., et al. 2002. Ventricular expression of natriuretic peptides in $\mathrm{Npr} 1(-/-)$ mice with cardiac hypertrophy and fibrosis. Am. J. Physiol. Heart Circ. Physiol. 283:H707-H714.

51. Knowles, J.W., et al. 2001. Pressure-independent enhancement of cardiac hypertrophy in natriuretic peptide receptor A-deficient mice. J. Clin. Invest. 107:975-984.

52. Nusz, D.J., et al. 2003. Vascular rarefaction in peripheral skeletal muscle after experimental heart failure. Am. J. Physiol. Heart Circ. Physiol. 285:H1554-H1562.

53. Dipla, K., Mattiello, J.A., Jeevanandam, V., Houser, S.R., and Margulies, K.B. 1998. Myocyte recovery after mechanical circulatory support in humans with end-stage heart failure. Circulation. 97:2316-2322.

54. Naga Prasad, S.V., Barak, L.S., Rapacciuolo, A., Caron, M.G., and Rockman, H.A. 2001. Agonistdependent recruitment of phosphoinositide 3-kinase to the membrane by beta-adrenergic receptor kinase 1. A role in receptor sequestration. J. Biol. Chem. 276:18953-18959. 